\title{
Offspring production of ovarian organoids derived from spermatogonial stem cells by chromatin reorganization
}

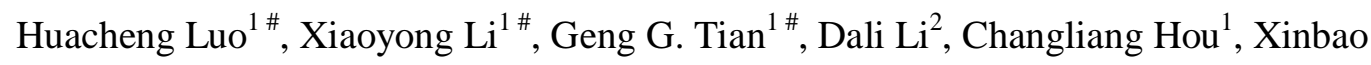

Ding $^{1}$, Lin Hou ${ }^{1}$, Qifeng Lyu ${ }^{3}$, Yunze Yang ${ }^{1}$, Austin J. Cooney ${ }^{4}$, Wenhai Xie ${ }^{1}$, Ji

Xiong ${ }^{1}, \mathrm{Hu} \mathrm{Wang}^{1}, \mathrm{Xiaodong}^{\mathrm{Zhao}}{ }^{5^{*}}, \mathrm{Ji} \mathrm{Wu}^{1,6,7^{*}}$

${ }^{1}$ Renji Hospital, Key Laboratory for the Genetics of Developmental \& Neuropsychiatric Disorders (Ministry of Education), Bio-X Institutes, School of Medicine, Shanghai Jiao Tong University, Shanghai 200032, China

${ }^{2}$ Shanghai Key Laboratory of Regulatory Biology, Institute of Biomedical Sciences and School of Life Sciences, East China Normal University, Shanghai, 200241, China.

${ }^{3}$ Shanghai Ninth People's Hospital Affiliated Shanghai Jiao Tong University Schoolof Medicine, Shanghai Jiao Tong University, Shanghai, 200240, China

${ }^{4}$ Department of Molecular and Cellular Biology, Baylor College of Medicine, Houston, TX 77030, USA

${ }^{5}$ Shanghai Center for Systems Biomedicine, Shanghai Jiao TongUniversity, Shanghai 200240, China

${ }^{6}$ Key Laboratory of Fertility Preservation and Maintenance of Ministry of Education, Ningxia Medical University, Yinchuan 750004, China.

${ }^{7}$ Lead Contact

${ }^{\#}$ These authors contributed equally to this work.

Correspondence: jiwu@sjtu.edu.cn orxiaodongzhao@sjtu.edu.cn 
2 Abstract

3 Fate determination of germline stem cells remains poorly understood at the chromatin 4 structure level ${ }^{1,2}$. Here, we demonstrate successful production of offspring from 5 oocytes transdifferentiated from mouse spermatogonial stem cells (SSCs) with tracking of transplanted SSCs in vivo, single cell whole exome sequencing, and in 3D cell culture reconstitution of the process of oogenesis derived from SSCs. Furthermore, we demonstrate direct induction of germline stem cells (iGSCs) differentiated into functional oocytes by transduction of H19, Stella, and Zfp57 and inactivation of Plzf in SSCs after screening with ovarian organoids. Using high throughput chromosome conformation, we uncovered extensive chromatin reorganization during SSC conversion into iGSCs, which was highly similar to female germline stem cells. We observed that although topologically associating domains were stable during SSC conversion, chromatin interactions changed in a striking manner, altering $35 \%$ of inactive and active chromosomal compartments throughout the genome. These findings have important implications in various areas including mammalian gametogenesis, genetic and epigenetic reprogramming, biotechnology, and medicine.

\section{Main}

Cell fate decisions, which require key gene regulation, remain poorly understood at the chromatin structure level. Although three-dimensional chromatin architectures of mouse gametes were recently reported, how they affect fate decisions of germline stem cells remains to be explored ${ }^{1-3}$.

To characterize spermatogonial stem cells (SSCs), we firstly isolated by magnetic activated cell sorting (MACS) using an anti-Thy-1 antibody after two step enzymatic digestion of the testes from 6-day-old pou5f1 (also known as Oct4, a germ cell-specific transcriptional factor)/GFP transgenic $\times$ C57BL/6 F1 hybrid mice ${ }^{4}$. Then, the isolated SSCs were purified for GFP-positive SSCs by fluorescence-activated cell sorting (FACS) (Extended Data Fig. 1a). The purified SSCs were maintained on SIM mouse embryo derived thioguanine- and ouabain- resistant (STO) feeder layers (see METHODS) (Extended Data Fig. 1b). After 3-5 days of culture, SSCs expanded into clusters (Extended Data Fig. 1c). Next, we determined the expression patterns of Oct4, 
$1 M v h$ (mouse vasa homologue, expressed exclusively in germ cells), $c$ - $R e t^{5}, P l z f^{6}$, $2 \quad \operatorname{Rex}-1^{7}, U t f 1^{8}$, Esg-1 (also known as DPPA5) ${ }^{9}, \operatorname{Stra}^{10}{ }^{10}, \operatorname{Sox}^{11}$, and Nanog${ }^{12}$. Reverse 3 transcription-polymerase chain reaction (RT-PCR) and immunocytochemical analyses 4 showed that SSCs expressed Oct4, Mvh, c-Ret, Plzf, Rex-1, Utf1, Esg-1, and Stra8. 5 Cytogenetic analysis by treatment with colchicines followed by G-band staining 6 demonstrated a normal karyotype (40, XY) in the metaphase spreads of examined 7 SSCs (Extended Data Fig. 1d-j). To verify the characterization of SSCs, we compared 8 the global expression profiles of SSCs and embryonic stem cells (ESCs) using 9 microarrays. Gene expression profiles by scatter plots showed a significant difference genes (2251) were differentially expressed between SSCs and ESCs, including pluripotency-related genes Dppa4, Fgf4, Nanog, Sox2, and Klf4 (Extended Data Fig. 1k, 1, fold change $>2, \mathrm{P}<0.05$, t-test) and SSC-related genes Zbtb16(or Plzf), Gfral, Tex18, Piwil2, and Dazl (Extended Data Fig. 1k, 1, fold change $>2, \mathrm{P}<0.05$, t-test). Therefore, these results demonstrated that SSCs had their apparent original property rather than a pluripotent identity. To determine the imprinting pattern of SSCs, differentially methylated regions (DMRs) of two paternal (H19 and Rasgrfl) and two maternal (Igf2r and Peg 10) imprinted regions were examined in SSCs and ESCs by bisulfite genomic sequencing. In SSCs, paternally imprinted regions (Extended Data Fig. $1 \mathrm{~m}$, o) were methylated, while maternally imprinted regions were not methylated (Extended Data Fig. 1n, p); this indicated an androgenetic imprinting pattern that was different from that of ESCs.

For investigating SSC fate determination in the mouse ovary, pou5f1/GFP transgenic mouse SSCs cultured for 3-5 days were directly transplanted into the ovaries of premature ovarian failure (POF) mice (see METHODS). Phosphate buffered saline (PBS) was injected into the ovaries of POF recipients as a control. For the positive control, female germline stem cells (FGSCs) from pou5f1/GFP transgenic mice were also transplanted into the ovaries of POF mice (see METHODS). At 8 weeks post-transplantation, recipient ovaries including positive control ovaries were collected and evaluated for morphology and GFP expression. Histological analysis showed that recipient ovaries injected with cells contained numerous oocytes at all stages of development, including GFP-positive oocytes (Fig. 1a I, III, IV and 
1 Extended Data Fig. 2a-e). Furthermore, DNA fluorescence in situ hybridization (FISH)

2 analysis showed the presence of the Sry gene in oocytes from recipient ovaries (Fig.

3 1b). For confirmation, single cell whole exon sequencing was used. The results

4 demonstrated that the germinal vesicle $(\mathrm{GV})$ oocytes from recipient ovaries were

5 derived from transplanted SSCs (Fig. 1c). Mature oocytes from recipient ovaries were

6 then collected for karyotype analysis. The results showed that some mature oocytes

7 had the karyotype of 20, Y (Fig. 1d I-V). PCR analysis of DNA fragment Sry

8 confirmed that some mature oocytes contained a candidate of the Y chromosome (Fig.

9 1d VI). However, control ovaries consisted of stromal and interstitial cells as well as atretic follicles (Fig. 1a II). These results indicate that XY oocytes were regenerated in POF females by transplantation of SSCs.

To examine whether XY oocytes derived from SSCs could produce offspring, POF recipients were mated with wild-type C57BL/6 adult males at 35 days after cell transplantation or PBS injection (control) $)^{13,14}$. Control recipients were not fertile $(n=$ 9). All POF recipients produced offspring $(n=8$, Fig. 1e I) with more males than females per litter (male:female, 1.95:1.00). One hundred and sixteen of the 130 offspring were alive with a normal phenotype as well as fertile. Fourteen of the 130 offspring died at 1-6 weeks after birth. The offspring were examined for the presence of GFP transgenes by Southern blot analyses (Fig. 1e II). Sixty-four of the 130 F1 progeny were heterozygous for the GFP transgene. Furthermore, simple sequence length polymorphism (SSLP) analysis was performed with SSLP markers to confirm that the offspring were derived from transplanted SSCs. The offspring from eight recipients (see above) were distinct from POF (see METHODS) or C57BL/6 mice-their parents (POF mice and mated male); however, they had exactly the same profiles as the SSCs from which they were derived (Fig. 1f). After analysis of the methylation status, five of the 20 offspring demonstrated abnormal methylation patterns. Two offspring that did not survive showed high methylation in Peg 10 with an increase of $21.46 \% \pm 2.66 \%$ and $28.16 \% \pm 2.87 \%$ compared with the control. The remaining three demonstrated that $H 19$ was highly methylated and increased by $16.66 \% \pm 1.48 \%$ and $14.23 \% \pm 1.38 \%$ or had low methylation with a decrease of 
$1 \quad 14.94 \% \pm 1.26 \%$ compared with the control. Moreover, five out of six positive controls

2 (see METHODS) with FGSC transplantation were fertile with approximately equal

3 numbers of males and females per litter (male:female, 1.02:1.00), and their offspring

4 showed no abnormal phenotype. Fifty of the 101 offspring were heterozygous for the

5 GFP transgene (Extended Data Fig. 2f, g). Although approximately $11 \%$ of the

6 offspring were abnormal, these results suggest that the XY oocytes derived from SSCs

7 can produce offspring in previously sterile recipients and generate transgenic progeny.

8 Control adult mice that received PBS injections into their ovaries did not produce

9 transgenic offspring.

For understand how the SSCs transdifferentiated into oocytes in recipient ovaries, pou5f1/GFP transgenic mouse SSCs cultured for 3 days were directly transplanted into the ovaries of POF mice, and then monitored by confocal laser scanning microscopy. At 2 hours after SSC transplantation, the SSCs were observed in ovaries of recipient mice, indicating that the SSCs had been successfully transplanted into the mouse ovary (Fig. 2a). The transplanted cells were found to migrate toward the edge of the ovarian cortex at 2 days post-transplantation (Fig. 2a). At 3 days after transplantation, the cells continuously migrated toward the edge of the ovarian cortex and some of them reached the edge (Fig. 2a). When the transplanted cells had been in the ovary for 4 days, all of them had migrated into the edge of the ovarian cortex (Fig. 2a). Five days post-transplantation, transplanted cells settled in the edge of the ovarian cortex and began to transdifferentiate into early primary oocytes (Fig. 2a, b). At 6-15 days after transplantation, the transplanted cells continued to transdifferentiate into oocytes at various stages of development (Fig. 2a). This was confirmed by dual immunofluorescence analysis of the expression of MVH and GFP in transplanted cells (Fig. 2c).

To explore the mechanism of SSC fate determination when the SSCs were transplanted into the recipient ovary, we performed bisulfite sequencing to analyze the methylation status of these transplanted cells, mainly the differentially methylated regions (DMRs) of paternally imprinted gene H19 and maternally imprinted gene Peg10. It is noteworthy that no obvious change of methylation levels, including the 
1 maternally or paternally imprinting gene, was observed at 2 hours after SSC 2 transplantation (Extended Data Fig. 3a-d). At 3-4 days after transplantation, 3 methylation levels of $H 19$ were reduced gradually to $68.3 \%$ and $60.8 \%$, with a further 4 reduction to $37.9 \%$ and $23.8 \%$ at 5-6 days, suggesting that the bulk of methylation 5 erasure occurred at 5-6 days. The low levels of methylation were present at 9 days and persisted to 15 days (Extended Data Fig. 3a, c). In contrast, the maternally imprinted gene PeglO showed evidence of robust de novo methylation with an increase to $45.7 \%$ methylation at 3 days and further increase to $78 \%$ methylation at 6 day, indicating that the bulk of methylation establishment occurred at 6 days. The high methylation levels were maintained from 9 to 15 days (Extended Data Fig. 3b, d). These results suggested that the maternal DNA methylation pattern was directly constructed during SSC development in recipient ovaries, and that the SSCs did not dedifferentiate into PGCs.

Furthermore, we determined expression patterns of imprinted genes (H19, Grb10, Gtl2, Rasgrf1, Peg10, Igf2r, and Snrpn) and important transcription factor genes (Plzf, Stella or Dppa3, Zfp42, Zfp57, and Nanos2) during transdifferentiation of the transplanted cells into oocytes in recipient ovaries based on data from our previous studies ${ }^{15,16}$. After comparing the expression of imprinted genes in the cultured SSCs (0 hour) and transplanted SSCs at 2 hours to 15 days post-transplantation, we observed that paternally imprinted genes (H19, Grb10, Rasgrfl, and Gtl2) and transcription factor genes (Stella and ZFP57) were gradually upregulated, especially at 3 and 6 days with a further increase from 9 to 15 days (Extended Data Fig. 3e). In contrast, along with transdifferentiation into oocytes of the SSCs in recipient ovaries, the expression levels of these maternally imprinted genes (Peg 10, Igf $2 r$, and Snrpn) and transcription factor genes (Plzf, Zfp42, and Nanos2) underwent obvious reductions at 5-6 days with a continuous decrease from 9 to 15 days after transplantation (Extended Data Fig. 3e), suggesting establishment of the maternal imprinting pattern.

Based on the above results, we further screened for the critical imprinted genes and transcription factor genes required for SSC conversion. The spatial organization of the mammal genome is known to play an important role in the regulation of gene 
1 expression $^{17}$. Therefore, we used in situ high throughput chromosome conformation 2 capture (Hi-C) to further screen for the critical genes and found 6.28 billion unique 3 read pairs in SSCs and FGSCs. The compartment status was divided into two groups, 4 compartment A and B. By comparing A/B compartment statuses and chromatin loops 5 between SSCs and FGSCs (Extended Data Fig. 4, Supplementary Table 1-3), we established combinations of six genes (6Gs), including imprinted genes, H19 and 7 Rasgrf1, and transcription factor genes Stella, Zfp57, Zfp42, and Plzf. After overexpressing Stella, H19, Zfp57, and Rasgrfl and knockdown of Plzf and Zfp42 in SSCs, the cells converted to induced germline stem cells (or induction of germline stem cells, iGSCs) with a maternal imprinted pattern (Extended Data Fig. 5, Extended Data Fig. 6a) and formed ovarian organoids when three-dimensional (3D) co-cultured with somatic cells from the fetal ovary for 2 weeks, modifying the method previously described in $\operatorname{ref}^{18}$ (Extended Data Fig. 6b I-II). Upon withdrawal of Rasgrfl from the $6 \mathrm{Gs}$, we found that the 3D co-cultured cells still formed ovarian organoids (Extended Data Fig. 6b III). For the remaining five genes (5Gs), removal of Zfp42 further promoted the formation of the organoids (Extended Data Fig. 6b IV). However, removal of any factor from the four genes (4Gs, Stella, H19, Zfp57, and Plzf) led to the failure to form the organoids (Extended Data Fig. 6b V-VIII).

The morphology of iGSCs induced by the overexpression of Stella, H19, and Zfp57 and the inactivation of Plzf was similar to that of FGSCs (Extended Data Fig. 7a). Furthermore, the iGSCs expressed Stella, Mvh, Fragilis, Dazl, and Oct4 with a maternal imprinted pattern (Extended Data Fig. 7a, b, Extended Data Fig. 6c). For confirmation, we performed genome-wide DNA methylation analysis in SSCs, iGSCs, and FGSCs by MeDIP-seq. A total of 38.7 million reads, yielding 467,163 DNA methylationsites (peaks) in three kinds of cell populations were generated. We observed widespread variation in terms of DNA methylation during SSC transition into iGSCs (Extended Data Fig. 7c). Subsequently, we performed pair-wide correlation analysis of the MeDIP data sets from SSCs, iGSCs, and FGSCs. We found that the overall DNA methylation pattern of iGSCs was similar to that of FGSCs $(r=$ $0.79)$, but it was less similar to that of SSCs $(r=0.59)$. Such a trend was evidenced 
1 more clearly by individual regions of interest. For example, at the maternally 2 imprinted region $\operatorname{Ig} 22 r$, the DNA methylation signal was relatively low in the $\operatorname{Ig} f 2 r$ 3 promoter of SSCs. It increased remarkably and appeared to be almost at the same 4 level in iGSCs and FGSCs (Extended Data Fig. 7c, d). A similar phenomenon was 5 also observed at the promoter region of $\mathrm{NrObl}$, the gene encoding the orphan nuclear 6 receptor and required for development of male characteristics in mice ${ }^{19}$. These 7 observations suggest that DNA methylation contributed to the SSC transition. Next, we compared global gene expression profiles among SSCs, iGSCs and FGSCs by 9 RNA sequencing. A total of 380,702,626 raw reads were generated. We detected expression of 18229, 19755, and 18978 out of 24550 genes in SSCs, iGSCs, and FGSCs, respectively. On average, $77 \%$ of the known mouse genes were expressed in the sampled SSCs, iGSCs, and FGSCs. Hieratical clustering was performed, and the results indicated that iGSCs were clustered with FGSCs, but separated from SSCs, suggesting that the global gene expression profile of iGSCs was similar to that of FGSCs (Extended Data Fig. 7e, f). Among these genes, Igf $2 r$, a maternal imprinted gene, showed high expression in SSCs and low level expression in iGSCs and FGSCs (Extended Data Fig. 7f), which was consistent with the results from the analysis of genome-wide DNA methylation in SSCs, iGSCs, and FGSCs.

Hi-C interaction maps provide information on multiple hierarchical levels of genome organization ${ }^{20}$. To understand how genome organization is involved in SSCs transition to iGSCs, we also performed $\mathrm{Hi}-\mathrm{C}$ experiments using two biological replicates of iGSCs, generating a total of 2.96 billion unique read pairs. The Hi-C data analysis showed the high order chromatin organization of the whole genome in SSCs, iGSCs, and FGSCs (Fig. 3a-c, Extended Data Fig. 9). To examine the characteristics of their chromatin organization, we analyzed the pattern of compartment A/B in SSCs, iGSCs, and FGSCs. We found a large degree of spatial plasticity in the arrangement of the $\mathrm{A} / \mathrm{B}$ compartments or redistribution of the spatial organization of their genomes during SSC transition into iGSCs with $35 \%$ of the genome switching compartments. Furthermore, we found that the regions that changed their $\mathrm{A} / \mathrm{B}$ compartment status corresponded to a single or series of topologically associated domains (TADs), 
1 suggesting that TADs are the units of dynamic alterations in chromosome 2 compartments (Fig. 3b). Interestingly, we observed that iGSCs and FGSCs were 3 highly similar in their status of A/B compartments compared with SSCs (Fig. 3c). For 4 SSC transition into iGSCs, genes that changed from compartment B to A tended to 5 show higher expression, while genes that changed from $\mathrm{A}$ to $\mathrm{B}$ tended to show 6 reduced expression (Fig. 3d). Moreover, we identified 4353 genes with co-variation 7 between compartment switching and gene expression. For example, at the compartment B region, Dppa3 expression was relatively low in SSCs. It increased remarkably and appeared to be almost at the same level in iGSCs and FGSCs when changing from compartment B to A (Fig. 3e). DNA methylation that changed from compartment B to A also tended to show a reduced signal, whereas DNA methylation that changed from A to B tended to show higher signal (Fig. 3f). Take together, these results demonstrate that, when SSCs convert to iGSCs, there is a high degree of plasticity in A and B compartments, corresponding changes in gene expression, indicating that the A and $\mathrm{B}$ compartments have a contributory to cell type-specific patterns of gene expression.

Developmental feature of ovarian organoids from FGSCs and iGSCs were explored. At 2 weeks of 3D co-culture with the germline stem cells and somatic cells from the fetal ovary, ovarian organoids were generated in FGSC and iGSC groups. The organoids were completely filled with follicles which possessed oocytes. In contrast, ovarian organoids were not formed in the SSC group. When the ovarian organoids were 3D co-cultured for 3 weeks, the follicles grew obviously (Fig. 4a). After these follicles were 3D cultured individually for 2 weeks (see METHODS), a large number of immature oocytes were obtained from the cultured follicles. Moreover, Oct4-EGFP cells were detectable in iGSC and FGSC groups at 3 days of 3D co-culture. A number of EGFP-positive oocytes were observed in ovarian organoids after 3 weeks of 3D co-culture (Fig. 4a). After 2-3 weeks of 3D co-culture, however, the EGFP expression became weak (Fig. 4a).

For comparison between iGSC and FGSC groups, 905 immature oocytes from the iGSC group were obtained from 27 ovarian organoids in 8 cultures with 
$133.52 \pm 4.27$ immature oocytes per organoid. In the FGSC group, 1140 immature

2 oocytes were obtained from 36 ovarian organoids in 12 experiments with $31.67 \pm 3.86$

3 immature oocytes per organoid. No difference in immature oocytes per organoid was

4 observed in both groups (Fig. 4b).

5 To evaluate oogenesis during the ovarian organoid development, 6 immunofluorescence analysis of SCP3 and H2AX was performed. Furthermore, the 7 gene expression profiles during iGSC or FGSC differentiation were analyzed by 8 qRT-PCR. The results showed progression of meiotic prophase I from 7 to 21 days of 9 the germ cell differentiation in both groups (Fig. 4c). The expression dynamics of genes involved in oogenesis were similar between both groups (Extended Data Fig. 11 10). oocytes reached mature oocytes in iGSC and FGSC groups, respectively. In addition, no difference in the number of mature oocytes was observed between the two groups (Fig. 4d, Extended Data Fig. 11a).

To determine whether these mature oocytes could develop into offspring following in vitro fertilization, embryo culture and transfer into pseudopregnant ICR females were performed. The fertilization rate of the iGSC group (47.2\%) was similar to that of the FGSC group (49.9\%). Subsequently, these zygotes developed to 2-cell embryos (Fig. 4e, Extended Data Fig. 11b). After the embryo transfer, 53 (male:female, 1.65:1.00) out of 342 (for iGSC group) or 51 (male:female, 1.13:1.00) out 355 (for FGSC group) were delivered as viable offspring with colored eyes (Fig. 4f, Extended Data Fig. 11c), indicating that the offspring were derived from C57BL/6 iGSC- or FGSC-derived oocytes, but not ICR oocytes among gonadal somatic cells. The offspring were confirmed for the presence of GFP transgenes by Southern blot analysis, and live imaging by a Lumazone imaging system (Fig. 4g, h). All of the obtained offspring grew up normally and were fertile with no difference between the two groups. After analysis of the methylation status, 10 offspring per group demonstrated no observably abnormal methylation patterns (Extended Data Table 1). Our findings provide a new strategy to investigate stem cell biology, biotechnology, 
and medicine.

1. Battulin, N. et al. Comparison of the three-dimensional organization of sperm and fibroblast genomes using the Hi-C approach. Genome Biol16, 77 (2015).

2. Jung, Y. H. et al. Chromatin States in Mouse Sperm Correlate with Embryonic and Adult Regulatory Landscapes. Cell Rep18, 1366-1382 (2017).

3. Ke, Y. et al. 3D Chromatin Structures of Mature Gametes and Structural Reprogramming during Mammalian Embryogenesis. Cell170, 367-381 e320 (2017).

4. Yoshimizu, T. et al. Germline-specific expression of the Oct-4/green fluorescent protein (GFP) transgene in mice. Dev Growth Differ41, 675-684 (1999).

5. Naughton, C. K., Jain, S., Strickland, A. M., Gupta, A. \& Milbrandt, J. Glial cell-line derived neurotrophic factor-mediated RET signaling regulates spermatogonial stem cell fate. Biol Reprod $\mathbf{7 4}$, 314-321 (2006).

6. Costoya, J. A. et al. Essential role of Plzf in maintenance of spermatogonial stem cells. Nat Genet36, 653-659 (2004).

7. Xu, J., Sylvester, R., Tighe, A. P., Chen, S. \& Gudas, L. J. Transcriptional activation of the suppressor of cytokine signaling-3 (SOCS-3) gene via STAT3 is increased in F9 REX1 (ZFP-42) knockout teratocarcinoma stem cells relative to wild-type cells. J Mol Biol377, 28-46 (2008).

8. Okuda, A. et al. UTF1, a novel transcriptional coactivator expressed in pluripotent embryonic stem cells and extra-embryonic cells. EMBO J17, 2019-2032 (1998).

9. Tanaka, T. S. et al. Gene expression profiling of embryo-derived stem cells reveals candidate genes associated with pluripotency and lineage specificity. Genome Res12, 1921-1928 (2002).

10. Oulad-Abdelghani, M. et al. Characterization of a premeiotic germ cell-specific cytoplasmic protein encoded by Stra8, a novel retinoic acid-responsive gene. J Cell Biol135, 469-477 (1996).

11. Fong, H., Hohenstein, K. A. \& Donovan, P. J. Regulation of self-renewal and pluripotency by Sox2 in human embryonic stem cells. Stem Cells26, 1931-1938 (2008).

12. Chambers, I. et al. Functional expression cloning of Nanog, a pluripotency sustaining factor in embryonic stem cells. Cell113, 643-655 (2003).

13. Zou, K. et al. Production of offspring from a germline stem cell line derived from neonatal ovaries. Nat Cell Biol11, 631-636 (2009).

14. Wu, C. et al. Tracing and Characterizing the Development of Transplanted Female Germline Stem Cells In Vivo. Mol Ther25, 1408-1419 (2017).

15. Xie, W., Wang, H. \& Wu, J. Similar morphological and molecular signatures shared by female and male germline stem cells. Sci Rep4, 5580 (2014).

16. Li, X., Tian, G. G., Zhao, Y. \& Wu, J. Genome-wide identification and characterization of long noncoding and circular RNAs in germline stem cells. Sci Data6, 8 (2019).

17. Gorkin, D. U., Leung, D. \& Ren, B. The 3D genome in transcriptional regulation and pluripotency. Cell Stem Cell14, 762-775 (2014).

18. Hikabe, O. et al. Reconstitution in vitro of the entire cycle of the mouse female germ line. Nature539, 299-303 (2016).

19. Zhang, X. L. et al. Integrative epigenomic analysis reveals unique epigenetic signatures involved in unipotency of mouse female germline stem cells. Genome Biol17, 162 (2016).

20. Lieberman-Aiden, E. et al. Comprehensive mapping of long-range interactions reveals folding 


\section{METHODS}

Mice. C57BL/6, pou5f1-GFP transgenic mice [CBA-Tg (pou5f1-EGFP) 2Mnn] (The Jackson Laboratory) or pou5f1/GFP transgenic mice ${ }^{21} \times$ C57BL/6 F1 hybrid mice were used in this study. Premature ovarian failure (POF) Pten (phosphatase and tensin homolog deleted on chromosome 10$)^{\text {loxp/loxp }} ;$ Gdf9-Cre (Gdf9 promoter-mediated Cre recombinase $^{+}$) mice were produced and genotyped as described by Reddy et $a l^{22,23}$. POF $\left(\right.$ Pten $^{\text {loxp/loxp }} ;$ Gdf9-Cre $\left.{ }^{+}\right)$mice were used as recipients. Pten ${ }^{\text {loxp } / \text { loxp }}$ mice (B6.129S4-Pten $\left.{ }^{\mathrm{tm} 1 \mathrm{Hwu}}\right)$ and Gdf9-Cre [Tg (Gdf9-iCre)] mice were purchased from The Jackson Laboratory. Animal experimentation was approved by the Institutional Animal Care and Use Committee of Shanghai and performed in accordance with the National Research Council Guide for Care and Use of Laboratory Animals.

Isolation and culture of spermatogonial stem cells. Testes from 6-day-old pou5f1-GFP transgenic mice or pou5f1/GFP transgenic mice×C57BL/6 F1 hybrid mice were collected and decapsulated. Spermatogonial stem cells (SSCs) were isolated using methods described by $\mathrm{Wu}$ et $a l^{24,25}$ and Yuan et $a l^{26}$. The SSCs were purified by both magnetic activated cell sorting (MACS) with an anti-Thy-1 antibody and fluorescence activated cell sorting (FACS), according to the manufacturers' instructions. SSCs were cultured on mitotically inactivated SIM mouse embryo derived thioguanine- and ouabain- resistant $(\mathrm{STO})$ feeder cells $\left(5 \times 10^{4}\right.$ cells $/ \mathrm{cm}^{2}$; ATCC) in culture medium. For mitotic inactivation, STO cells were treated with 10 $\mu \mathrm{g} / \mathrm{ml}$ mitomycin C (Sigma) for 2-3 hours. Mitomycin C-treated STO cells were washed with phosphate buffered saline (PBS) and transferred to $0.2 \%$ (w/v) gelatin-coated tissue culture plates. The SSC culture medium consisted of high glucose Dulbecco's modified Eagle's medium (DMEM; Life Technologies) supplemented with $10 \%$ fetal bovine serum (FBS; GIBCO), $2 \mathrm{mM}$ L-glutamine (Sigma), $0.1 \mathrm{mM} \beta$-mercaptoethanol (Sigma), $1 \mathrm{mM}$ nonessential amino acids (Life Technologies), $10 \mathrm{ng} / \mathrm{ml}$ glial cell line-derived neurotrophic factor (GDNF; R\&D Systems), $10 \mathrm{ng} / \mathrm{ml}$ leukemia inhibitory factor (LIF; Chemicon), and $15 \mathrm{mg} / \mathrm{l}$ 
1 penicillin (Sigma). SSCs were cultured on STO feeders in 24-well plates with $500 \mu \mathrm{l}$

2 culture medium per well. The medium was replaced every 1-2 days, and cells were

3 subcultured at a split ratio of 1:1-3 by trypsinization every 3 days. All cultures were

4 maintained at $37^{\circ} \mathrm{C}$ with $5 \% \mathrm{CO}_{2}$.

5 Isolation and purification of female germline stem cells. Ovaries were collected

6 from 5-day-old pou5f1/GFP transgenic mice $\times$ C57BL/6 F1 hybrid mice. Female

7 germline stem cells (FGSCs) were isolated and purified using a method described

8 elsewhere ${ }^{27}$. Briefly, dissected ovarian tissues were incubated in $1 \mathrm{mg} / \mathrm{ml}$ collagenase

9 (type IV; Sigma) at $37^{\circ} \mathrm{C}$ with gentle agitation for 15-20 min. After washing, ovarian tissues were incubated in $0.05 \%$ trypsin and $1 \mathrm{mM}$ EDTA at $37^{\circ} \mathrm{C}$ for $5-7 \mathrm{~min}$. Sheep anti-mouse IgG magnetic beads (Dynal Biotech) were incubated with an anti-fragilis antibody (Abcam) for $30 \mathrm{~min}$ at room temperature. The magnetic bead/antibody mixture was incubated with the isolated cell suspension for another $30 \mathrm{~min}$ at room temperature. Then, the mixture of cells and magnetic beads was placed on a magnetic bead separator for 2-3 min, and the supernatant was removed. The fraction on the inner side of the eppendorf tube was collected and rinsed twice with PBS, resuspended in PBS, and further purified by FACS, in accordance with the manufacturers' instructions. The purified FGSCs were placed in FGSC culture medium $^{27}$ and cultured on mitotically inactivated STO feeder cells in 24-well plates at $37^{\circ} \mathrm{C}$ with $5 \% \mathrm{CO}_{2}$.

Preparation of ovarian tissue for analysis. Ovaries from recipient and control mice were fixed with $4 \%(\mathrm{w} / \mathrm{v})$ paraformaldehyde $\left(4^{\circ} \mathrm{C}\right.$, overnight $)$ and dehydrated via a graded ethanol series. The tissues were vitrified in xylene, embedded in paraffin, sectioned (6 $\mu \mathrm{m}$ thickness), and then mounted on slides. Prior to immunofluorescence staining, the sections were dewaxed in xylene and rehydrated via a graded ethanol series. Sections were counterstained with hematoxylin.

Immunofluorescence. After equilibration in PBS, tissue sections were digested with $0.125 \%$ trypsin for $10 \mathrm{~min}$ at $37^{\circ} \mathrm{C}$ and then washed in PBS twice. The sections were blocked in $10 \%$ goat serum at room temperature for $10 \mathrm{~min}$ and then incubated overnight at $4{ }^{\circ} \mathrm{C}$ with appropriate primary antibodies. The primary antibodies used were mouse monoclonal anti-GFP (1:200 dilution; Abcam) and rabbit polyclonal 
1 anti-MVH (1:200; Abcam). After washing in PBS, the sections were incubated at $237^{\circ} \mathrm{C}$ for $30 \mathrm{~min}$ with TRITC-conjugated goat anti-rabbit IgG (1:200; Sino-American 3 Biotechnology Co.) or fluorescein isothiocyanate (FITC)-conjugated goat anti-mouse $4 \quad \operatorname{IgG}(1: 200$; Sino-American Biotechnology Co.) as appropriate. Sections were stained 5 with 4',6-diamidino-2-phenylindole (DAPI, 1:1000) at $37^{\circ} \mathrm{C}$ for $20 \mathrm{~min}$, covered with 6 mounting medium (glycerol:PBS, 3:1), and viewed under a Nikon Eclipse E600 7 microscope equipped with a Nikon Dxm 1200 digital camera using fluorescein optics 8 for TRITC and FITC, and ultraviolet optics for DAPI or under a confocal microscope $9 \quad$ (FluoView ${ }^{\mathrm{TM}}$ FV1000). Cultured germline stem cells were fixed with $4 \%$ paraformaldehyde in PBS at room temperature for $20 \mathrm{~min}$. After fixation, the cells were permeabilized with $0.5 \%$ Triton X-100 for $30 \mathrm{~min}$ at room temperature for PLZF staining. The cells were incubated in blocking solution (10\% normal goat or bovine serum in PBS, $10 \mathrm{~min}, 37^{\circ} \mathrm{C}$ ), followed by rinsing and overnight incubation at $4^{\circ} \mathrm{C}$ with appropriate primary antibodies: rabbit polyclonal anti-MVH (1:200; Abcam), mouse monoclonal anti-GFP (1:200; Abcam), and anti-PLZF (1:150, Santa Cruz Biotechnology). After washing in PBS, the cells were incubated with TRITC-conjugated goat anti-rabbit IgG (1:200) or fluorescein isothiocyanate (FITC)-conjugated goat anti-mouse $\operatorname{IgG}(1: 150)$ at $37^{\circ} \mathrm{C}$ for $30 \mathrm{~min}$, rinsed, and then incubated with DAPI $(1: 1000)$ at $37^{\circ} \mathrm{C}$ for 20 min. Petri dishes were then covered with mounting medium (glycerol:PBS, 3:1) and viewed as described above.

Karyotypic analysis. Karyotypic analysis was performed using standard protocols for mouse chromosome analysis. After culture for 3 days, SSCs were treated with culture medium containing colchicine (100 ng/ml; Sigma) for 3 hours, hypotonically treated with $75 \mathrm{mM} \mathrm{KCl}$ for $15 \mathrm{~min}$ at $37^{\circ} \mathrm{C}$, immersed twice in methanol:acetic acid (3:1) for $30 \mathrm{~min}$ at $-30^{\circ} \mathrm{C}$, dried in airfor $3-4$ days, digested with $0.025 \%$ trypsin, and then stained with Giemsa. To verify the chromosomal type of recipient mouse oocytes, karyotypic analysis of mature oocytes from recipients was performed as describedpreviously ${ }^{28}$. To collect mature oocytes, recipient mice were superovulated with 10 IU pregnant mare serum gonadotropin (PMSG; ProSpec-Tany) for 48 hours, 
1 oocytes were hypotonically treated with $75 \mathrm{mM} \mathrm{KCl}$ at $37^{\circ} \mathrm{C}$ for $15 \mathrm{~min}$ and then 2 fixed with two solutions consisting of methanol/acetic acid/water (5:1:2) for 5-10 min 3 and methanol/acetic acid (3:1) for $15 \mathrm{~min}$ at room temperature. Fixed cells were 4 mounted on slides and immediately exposed to steam from boiling water $\left(90-100^{\circ} \mathrm{C}\right)$

5 for $30 \mathrm{sec}$ to cause expansion of the cells, followed by drying at $37^{\circ} \mathrm{C}$ and Giemsa 6 staining (Amresco) ${ }^{28}$.

7 Embryonic stem cell culture. Embryonic stem cells (ESCs) were cultured with 8 mouse embryonic fibroblasts in the presence of leukemia inhibitory factor (LIF; 1000 $\mathrm{U} / \mathrm{ml}$ ) in Glasgow modification of Eagle's medium (GMEM; Invitrogen) containing $10 \%$ fetal calf serum. The medium was replaced every 1-2 days, and cells subcultured at a split ratio of 1:1-3 by trypsinization every 3 days. All cultures were maintained at $37^{\circ} \mathrm{C}$ with $5 \% \mathrm{CO}_{2}$.

Microarrays. Total RNA was extracted from cultured SSCs and ESCs using Trizol reagent (Invitrogen), in accordance with the manufacturer's instructions. RNA was labeled using an Illumina labeling kit. An Illumina sentrix mouse WG-6 Beadchip (45281 transcripts) was used in this study. Microarray experiments, including RNA labeling, hybridization, washing, scanning, image analysis, normalization, and data processing, were performed by Shanghai Biotechnology Corporation using the Illumina manual. Three biological repeats were included in microarray experiments. Differentially expressed genes were identified by the Illumina system. The data were analyzed using GeneSpring GX 11 software. Hierarchical clustering of samples was performed by cluster 3.0 and TreeView software ${ }^{29}$.

Transplantation. For injection into the ovary, SSCs were collected and transplanted into the ovaries of POF mice. For the positive control, FGSCs from pou5f1/GFP transgenic mice were also transplanted into ovaries of POF mice. Recipient mice were anesthetized by injection of pentobarbital sodium ( $45 \mathrm{mg} / \mathrm{kg}$ ). Approximately $6 \mu \mathrm{l}$ of a singlecell suspension containing $1 \times 10^{4}$ cells or $6 \mu \mathrm{l}$ PBS for the control was microinjected into the ovaries of recipientsas described elsewhere. In detail, after anesthetization of recipient mice for 20-30 min and disinfection of the abdominal surface using $75 \%$ ethanol, the recipient abdominal cavity was carefully opened. To expose and find the ovaries, the intestines were carefully moved away from the inside of the abdominal cavity. The Y-shaped uterus was located, and then following the 
1 uterus and oviduct until posterior to the kidneys, the ovaries were located caudal to 2 the kidneys in the lower abdominal cavity. By gently holding an ovary with forceps 3 without causing damage, the ovary was injected at 1-2 sites using a glass pipette with $4 \quad$ a $45 \mu \mathrm{m}$ tip and mouth pipetting to carefully transplant the $6 \mu 1$ single cell suspension 5 of $\sim 1 \times 10^{4} \mathrm{SSCs}$ or FGSCs into each ovary. At 35 days after transplantation, 6 recipients were mated with 8 -week-old male mice.

7 Reverse transcription-polymerase chain reaction and Southern blotting. Reverse 8 transcription-polymerase chain reaction (RT-PCR), PCR, and Southern blotting were 9 performed as described elsewhere. Twenty-five cycles of PCR were performed using Taq polymerase (Takara) with primer sets specific for each gene. The glyceraldehyde-3-phosphate dehydrogenase gene (Gapdh) was amplified in each sample as a loading control. PCR products were isolated, subcloned, and sequenced to confirm the gene sequence.

Bisulfite genomic sequencing. Genomic DNA was extracted from SSCs, transplanted SSCs, ESCs, induced germline stem cells (iGSCs), and FGSCs. For bisulfite sequencing analysis of methylation, $500 \mathrm{ng}$ genomic DNA was processed using an EZ DNA Methylation-Gold Kit ${ }^{\mathrm{TM}}$ (ZYMO Research), in accordance with the manufacturer's instructions. The methylation status of imprinted genes was analyzed using primers (outside, 5'-GTTTTTTTGGTTATTGAAT-TTTAAAATTAGT-3' and 5'-AAAAACCATTCCGTAAATACACAAATACCTA-3', inside, 5'-TTAGTGTGGTTTATTATAGGAAGGTATAGAAGT-3' and 5'-TAAACCTAAAATACTCAAAACTTTATCACAA-3' for $H 19$; 5'-GTG TAG AAT ATG GGG TTG TTT TAT ATT G-3' and 5'-ATA ATA CAA CAA CAA CAA TAA CAA TC-3' for Rasgrf1; 5'-GTA AAG TGA TTG GTT TTG TAT TTT TAA GTG-3' and 5'-TTA ATT ACT CTC CTA CAA CTT TCC AAA TT-3' for Peg 10; 5'-TTA GTG GGG TAT TTT TAT TTG TAT GG-3' and 5'-AAA TAT CCT AAA AAT ACA AAC TAC ACA A-3' for Igf $2 r$; outside, 5'-TATGTAATATGATATAGTTTAGAAATTAG-3' and 5'-AATAAАCCCAAАTCTAАAАTATTTTAАTC-3', inside, 5'-AATTTGTGTGATGTTTGTAATTATTTGG-3' and 5'-ATAAAATACACTTTCACTACTAAAATCC-3' for Snrpn). PCR products were sequenced and $\mathrm{CpG}$ islands were analyzed.

PCR amplification of lineage-specific microsatellite loci. Genomic DNA was 
1 extracted from mouse tail tips or donor SSCs. DNA samples from donor SSCs, female

2 recipients, mated males, and their corresponding offspring were analyzed by simple

3 sequence length polymorphism (SSLP). Sequences for the primer pairs were designed

4 according to the Mouse Genome Informatics website

5 (http://www.informatics.jax.org/). Amplification of lineage-specific microsatellite

6 DNA was performed in accordance with a previously described procedure ${ }^{30}$. PCR

7 products were separated and analyzed by 3\% agarose gel electrophoresis (Bio-Rad)

8 and visualized by ethidium bromide staining.

9 Flow cytometry and cell sorting. After MACS, the cells were suspended in PBS and subjected to flow cytometry to analyze and sort GFP-positive cells using a FACSAria

11 II cell sorter equipped with BD software (Becton Dickinson).

Quantitative reverse transcription-PCR analysis. Total RNA from cells was isolated using Trizol reagent. Complementary DNA was synthesized from $2 \mu \mathrm{g}$ total RNA using a High Capacity cDNA Reverse Transcription Kit (Invitrogen). Primers were designed using Primer Premier Software (Primer Premier 5.0). Primer details are listed in Supplementary Table 4. Gapdh was amplified in each sample as an internal control. The mRNA level of each gene was normalized to Gapdh expression. The specificity of all quantitative real-time PCRs (qPCRs) was verified by a single peak in the melting curve. qPCRs were performed with a 7500 real-time PCR amplification system using SYBR Green PCR master mix (Applied Biosystems, UK). The relative levels of transcripts were calculated using the $\Delta \Delta \mathrm{CT}$ method within the $\mathrm{ABI} 7500$ System Software (V2.0.4). All gene expression levels were normalized to the internal standard gene, Gapdh. The means and standard error were calculated from triplicate measurements. Significance was determined using the Student's $t$-test. A $P$-value of less than 0.05 was considered as significant, and a $P$-value of less than 0.01 was extremely significant.

Single cell whole genome amplification and exome sequencing. Single cell whole genome amplification was performed on lysed single cells using a recently developed method named multiple annealing and looping based amplification cycles

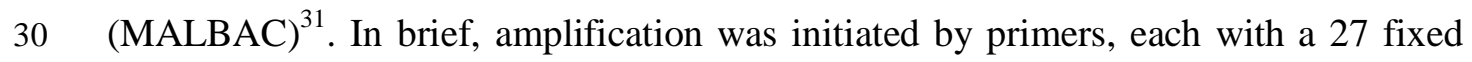


1 and eight degenerate base hybridizing uniformly throughout the genome. Fragments

2 with variable length at random starting positions were generated by polymerase 3 extension for multiple cycles. All fragments were flanked by the 27 base-fixed 4 sequence and their complementary sequences, and further amplified by PCR to about $5 \quad 1 \mu \mathrm{g}$ for barcoded massively parallel sequencing on an Illumina HiSeq 2500 6 sequencing platform.

7 Sry DNA in situ hybridization. We used a commercially available SRY DNA FISH kit (Mice SRY DNA biotin labelled POD and fluorescent FISH in situ hybridization 9 double staining system, TBD Science), according to the manufacturer's instructions. Briefly, sections were dewaxed with a graded series of ethanol, quenched in $3 \% \mathrm{H}_{2} \mathrm{O}_{2}$ for $10 \mathrm{~min}$ at room temperature, and then washed twice with PBS. The sections were covered with SRY reagent B for 10 min at $37^{\circ} \mathrm{C}$. After washing with PBS, the sections were incubated in Tris buffered saline (TBS) for $20 \mathrm{~min}$ at $95-100^{\circ} \mathrm{C}(\mathrm{pH} 8.9)$ and then rinsed three times with cold TBS ( 5 min per rinse) and once with $0.2 \times$ saline sodium citrate ( $5 \mathrm{~min}$ per rinse) at $0{ }^{\circ} \mathrm{C}$. The sections were incubated for 8 hours at $37^{\circ} \mathrm{C}$ with SRY reagent A and then washed three times with $2 \times$ saline sodium citrate at $37^{\circ} \mathrm{C}$ ( 3 min per rinse), three times with $0.2 \times$ saline sodium citrate ( $3 \mathrm{~min}$ per rinse) at $37^{\circ} \mathrm{C}$, and three times with TBS $(2 \mathrm{~min}$ per rinse $)$ at $37^{\circ} \mathrm{C}$. The sections were covered with SRY reagent $\mathrm{C}$ for $45 \mathrm{~min}$ at $37^{\circ} \mathrm{C}$. After washing in $\mathrm{PBS}$, the sections were incubated at $37^{\circ} \mathrm{C}$ for $120 \mathrm{~min}$ with a fluorescein isothiocyanate (FITC)-conjugated mouse anti-digoxin monoclonal antibody, then incubated at $37^{\circ} \mathrm{C}$ for 120 min with DAPI. Finally, the sections were mounted in anti-fade mounting medium. Images were obtained using a Leica DMI3000 B microscope and Leica DFC550 digital camera.

In situ high throughput chromosome conformation capture library generation using a low amount of cells. In situ high throughput chromosome conformation capture (Hi-C) assays were carried out according to the protocol with minor modifications ${ }^{32-34}$. Cells were fixed in a $1 \%$ final concentration of formaldehyde prior to $10 \mathrm{~min}$ incubation at room temperature. The reaction was quenched for $5 \mathrm{~min}$ by adding a $2.5 \mathrm{M}$ glycine solution. Cells were pelleted twice $\left(3000 \mathrm{~g}, 4^{\circ} \mathrm{C}\right.$ for $\left.5 \mathrm{~min}\right)$, 
1 resuspended in ice-cold $\mathrm{Hi}-\mathrm{C}$ lysis buffer for at least $15 \mathrm{~min}$, and then washed once 2 with $100 \mu$ of $1 \times$ NEBuffer 2 . The supernatant was discarded, and $1 \mu$ of $5 \%$ sodium 3 dodecyl sulfate (SDS) was added to the remaining $9 \mu$ l solution. The pellet was gently 4 mixed and incubated at $62^{\circ} \mathrm{C}$ for $10 \mathrm{~min}$. After incubation, $9.5 \mu \mathrm{l}$ water and $2.5 \mu \mathrm{l}$ of $510 \%$ Triton X-100 were added to quench the SDS, and then the solution was 6 incubated at $37^{\circ} \mathrm{C}$ for $30 \mathrm{~min}$. Chromatin digestion was performed with Dpn II 7 restriction enzyme (NEB, R0543M) at $37^{\circ} \mathrm{C}$ overnight and then inactivated for $20 \mathrm{~min}$ mix of $3.75 \mu \mathrm{l}$ biotin-14-dATP (Life Technologies), $0.45 \mu \mathrm{l}$ of $10 \mathrm{mM}$ $\mathrm{dCTP} / \mathrm{dGTP} / \mathrm{dTTP}$ mix, and $1 \mu \mathrm{l}$ of $5 \mathrm{U} / \mu \mathrm{l}$ large DNA Polymerase I (NEB, M0210L) were added,followed by incubation at $24^{\circ} \mathrm{Cfor} 4$ hours. The above biotin-labelled products were ligated by adding a master mix of $66.3 \mu \mathrm{l}$ water, $12 \mu \mathrm{l}$ of 10× NEB T4 DNA ligase buffer, $10 \mu \mathrm{l}$ of $10 \%$ Triton $\mathrm{X}-100,5 \mu \mathrm{l}$ of $10 \mathrm{mg} / \mathrm{ml}$ bovineserum albumin, and $2 \mu \mathrm{l}$ of $400 \mathrm{U} / \mathrm{ml} \mathrm{T} 4 \mathrm{DNA}$ ligase, followed by incubation at $16^{\circ} \mathrm{C}$ for 20 hours and then inactivation at $75^{\circ} \mathrm{C}$ for $20 \mathrm{~min}$. The samples were pelleted $\left(3000 \mathrm{~g}, 4^{\circ} \mathrm{Cfor} 5 \mathrm{~min}\right)$ and washed once with $100 \mu \mathrm{l}$ of $10 \mathrm{mM}$ Tris buffer. To remove biotin from unligated DNA ends, a master mix of $40 \mu \mathrm{l}$ water, $5 \mu \mathrm{l}$ of $10 \times$ NEBuffer $2.1,0.125 \mu \mathrm{l}$ of $10 \mathrm{mM}$ $\mathrm{dATP} / \mathrm{dGTP}$, and $5 \mu \mathrm{l}$ of 3,000 U/ml T4 DNA polymerase (NEB, M0203L) were added to the tube containing the DNA sample, followed by incubation at $20^{\circ} \mathrm{C}$ for 4 hours. The samples were pelleted $\left(3000 \mathrm{~g}, 4^{\circ} \mathrm{Cfor} 5 \mathrm{~min}\right)$ and resuspended in $50 \mu \mathrm{l}$ of $10 \mathrm{mM}$ Tris buffer. To digest the proteins, $2 \mu \mathrm{l}$ of $20 \mathrm{mg} / \mathrm{ml}$ proteinase $\mathrm{K}$ (NEB, P8107S) was added, followed by incubation at $62^{\circ} \mathrm{C}$ for 18 hours and inactivation at $75^{\circ} \mathrm{C}$ for $30 \mathrm{~min}$. The DNA was sheared to an average size of $400 \mathrm{bp}$ (Covaris, M220) to perform the End Repair/dA-Tailing and Adaptor Ligation (NEB, E7337A) with a KAPA Hyper Prep Kit (KAPA, kk8502) and then processed by $3 \mu$ of USER ${ }^{\mathrm{TM}}$ Enzyme (NEB, M5505L) at $37^{\circ} \mathrm{C}$ for $15 \mathrm{~min}$ to open up the loop. Biotin-labeled ligation products were isolated using MyOneStreptavidin T1 Dynabeads (Life Technologies, 65601) and then resuspended in $20 \mu \mathrm{l}$ of $10 \mathrm{mM}$ Tris buffer at $98^{\circ} \mathrm{C}$ for $10 \mathrm{~min}$, and the supernatant was transferred to a fresh PCR tube. Hi-C DNA was amplified using Index Primers set 1 (NEB, E7335S). The Hi-C libraries were purified 
1 with AMPure XP beads (Beckman Coulter, A63881) and sequenced using an Illumina 2 sequencing platform.

3 Hi-C data processing, mapping, and ICE normalization. For Hi-C pair-end raw 4 data, we first trimmed the adaptor sequences and low quality reads with BBmap 5 (version 38.16). Then, we used HiCPro (version 2.7) (5) $^{35}$ to map, process, and perform 6 iterative correction for normalization. Briefly, reads were independently aligned to the 7 mouse reference genome $(\mathrm{mm} 9)$ by the bowtie 2 algorithm ${ }^{36}$. We discarded the uncut DNA reads, re-ligation reads, continuous reads, and PCR artifacts. We then used the unique mapped reads (MAPQ>10) to build the contact matrix. Valid read pairs were then binned at a specific resolution by dividing the genome into sequential bins of equal size. We generated the raw contact matrices at binning resolutions of 10, 20, 40, 100 , and $200 \mathrm{~kb}$. ICE ${ }^{37}$ normalization was applied to remove bias in the raw matrix, such as GC content, mappability, and effective fragment length in the Hi-C data.

Validation of Hi-C data. The data reproducibility was confirmed by calculating Pearson's correlation coefficient (PCC) between the two libraries. Briefly, the interaction frequency was generated for each pair of $40 \mathrm{~kb}$ bins. For each possible interaction $\mathrm{I}_{\mathrm{ij}}$ between two replicates, they were correlated by comparing each point interaction in the normalized interaction matrix. Considering that the interaction matrix was highly skewed toward proximal interactions, we restricted the correlation to a maximum distance of $2 \mathrm{Mb}$ between points $\mathrm{i}$ and $\mathrm{j}$. We used $\mathrm{R}$ to calculate Pearson's correlation between two duplicates.

Contact probability $\boldsymbol{p}(s)$ calculation. $P(s)$ was calculated with normalized interaction matrices at a $40 \mathrm{~kb}$ resolution, as describedpreviously ${ }^{30}$. $P(s)$ calculations only considered intra interactions. Briefly, we divided the genome into $40 \mathrm{~kb}$ bins. For each distance separated by $40,80,120$, and $160 \mathrm{~kb}$, we counted the number of interactions at corresponding distances. Then, we divided the number of interactions in each bin by the total number of possible region reads as $P(s)$. Furthermore, we normalized the sum of $P(s)$ over the range of distances as 1 . We used LOWESS fitting to construct the curve (log-log axis).

30 Identification of $\mathbf{A}$ and $\mathbf{B}$ compartments. We used the R package (HiTC) ${ }^{38}$ pca.hic 
1 function to generate PC1 eigenvectors using 400kb normalized matrices with the

2 following options: normPerExpected=TRUE, $\mathrm{npc}=1$, for which a positive value

3 indicated the A compartment, while a negative value indicated the B compartment. To

4 investigate compartment switching, we defined switched bins only if PC1

5 eigenvectors changed in the same direction for two replicates.

6 Identification of concordant genes with an A/B compartment switch. We used a

7 previously described method with minor modifications to define genes with

8 concordant changes in expression and compartment status ${ }^{39}$. Briefly, we calculated the

9 covariance between the vector of the gene expression values (FPKM) and the vector

10 of PC1 values for each gene across five cell types. The calculated covariance as a

11 metric to quantitatively define "concordance" was used. We compared these observed

12 covariance values with a random background distribution to calculate a $\mathrm{P}$-value for

13 the covariance for each gene. Then, we produced the background distribution by

14 randomly shuffling the vector of FPKM for each gene and calculating the covariance

15 between the PC1 values and random gene expression vector. A rank-based P-value

16 could be calculated for observed covariance values with 1000 repeats for each gene.

17 Concordant genes were defined as those with a P-value of $<0.01$.

18 Direct induction of germline stem cells from SSCs. Knockdowns of specific genes

19 were accomplished by small interfering RNAs (siRNAs) targeting Plzf and Eed. The

20 interfering fragment was inserted downstream of the U6 promoter in a lentiviral vector (pLKD-CMV-G\&PR-U6-shRNA) by molecular biological methods. At least four independent siRNAs were screened for knockdown efficiency against each target and the best siRNA target was selected (target Seq: CCAGGCATCTGATGACAAT for Plzf; GCAACAGAGTAACCTTATA for Eed). For Stella, Zfp57, H19, and Rasgrf1 overexpression, cDNAs of candidate genes were inserted into the EcoRI and BamHI restriction sites of the overexpression plasmid (pHBLV-CMVIE-ZsGreen-T2A-puro). Lentivirus particles were generated by cotransfection of knockdown or overexpression 28 plasmids and lentivirus packaging plasmids into HEK293T cells using transgene reagent. Enhancing buffer was added to the medium after 12 hours of transfection. 
1 Virus particles were harvested at 48 hours after transfection, and a standardized virus

2 titer was obtained using HEK293T cells.

3 For lentivirus infection, $1 \times 10^{4} \mathrm{SSCs}$, which were passaged for 2-3 times, were seeded

4 in the well of a 48-well plate pre-coated with laminin and incubated with a 1:1

5 mixture of culture medium and lentivirus-concentrated solution (lentivirus titer:

$6 \quad 1 \times 10^{9} \mathrm{TU} / \mathrm{ml}$ ) containing $5 \mu \mathrm{g} / \mathrm{ml}$ polybrene. After overnight infection, cells were

7 re-plated onto puromycin-resistant STO feeder layers and cultured in SSC medium. At

812 hours after re-plating, the SSCs were incubated with a 1:1 mixture of culture

9 medium and lentivirus-concentrated solution again. After overnight infection, the mixture was changed to fresh culture medium, and the cells were cultured for 12 hours. SSCs were then infected for a third time. After overnight infection, the mixture was changed to fresh culture medium, and the cells were cultured at $37^{\circ} \mathrm{C}$ with $5 \%$ $\mathrm{CO}_{2}$. At day 6, the cells were subcultured at a 1:1-2 split ratio, and $100 \mathrm{ng} / \mathrm{ml}$ puromycin was added to the FGSC culture medium to screen for puromycin-resistant iGSCs. After 72 hours, the surviving iGSCs were passaged and analyzed by qRT-PCR and western blotting.

RNA-seq library generation and data analysis. Total RNA was extracted from $1-2 \times 10^{6}$ cells using Trizol Reagent. The RNA quality was assessed using an Agilent Bioanalyzer 2100. RNA-Seq libraries were prepared using the KAPA Stranded mRNA-Seq kit, following the manufacturer's instructions. After preparation, libraries were quantified using a Qubitfluorometer and sequenced with the HiSeq Platform ( $2 \times 100 \mathrm{bp}$ ). All RNA-Seq data were trimmed and aligned to the $\mathrm{mm} 9$ reference genome using Hisat2 (version 4.8.2) ${ }^{40}$ with the default parameters. Gene expression as FPKM was calculated by Cufflinks (version 2.2.1) ${ }^{41}$ using the RefSeq database from the UCSC genome browser. Sequencing depth was normalized.

GO term enrichment analysis. GO term enrichment analysis was performed using the DAVID tool (version 6.8) ${ }^{42}$, focusing on enriched biological processes (BP). The GO results were displayed by Cytoscape (version 3.5.1) ${ }^{43}$. For the Benjamin-corrected P-value, a threshold of $<0.05$ was used for significance. 
1 MeDIP-seq and bioinformatics. The DNA methylome assay was performed as 2 described previously ${ }^{44}$. Briefly, genomic DNA (gDNA) was extracted and fragmented 3 with Bioruptor (Connecticut, USA) into fragment sizes of 200-500 bp. Sonicated 4 gDNA was used for end-repair and adaptor ligation. The adaptor-ligated gDNA was 5 denatured and incubated with an antibody (Epigentek, A-1014) conjugated on Protein 6 A+G Magnetic beads (Millipore, 16-663). Immunoprecipitated DNA was amplified 7 by PCR and subjected to Illumina sequencing.

MeDIP and input raw sequencing reads were mapped using Bowtie2 (version 2.2.6) to the UCSC mm10 genome reference ${ }^{36}$. Duplicate reads were removed by samtools (version: 1.6-1). The normalized coverage was calculated by binning the unique tags in $1 \mathrm{~kb}$ bins, and the number of reads in each bin was normalized using reads per kilobase per million reads (RPKM). We identified the enriched MeDIP regions over the background with MACS (version 2.1.1) and default parameters ${ }^{45}$. Genome-wide pairwise correlation analysis of read depth in $1 \mathrm{~kb}$ bins was performed to evaluate DNA methylation patterns of SSCs, iGSCs, and FGSCs.

Ovarian organoid generation and culture. Ovarian organoids were formed using a modified method described elsewhere ${ }^{46}$. Briefly, iGSCs, FGSCs (positive control), and SSCs (negative control) were purified by a FACS Aria II (BD Bioscience) and co-cultured with E12.5 female gonadal somatic cells in a 96-well U-bottom, low-binding culture plate (Thermo Fisher Scientific) for 2 days in GMEM supplemented with 15\% Knockout serum replacement (Invitrogen), $1 \mu \mathrm{M}$ retinoic acid, 2 mM L-glutamine (Sigma), $1 \mathrm{mM}$ non-essential amino acids (Life Technologies), $2 \mathrm{mM}$ L-glutamine (Sigma), $30 \mathrm{mg} / \mathrm{ml}$ pyruvate (Amresco), 50 $\mathrm{mM} \beta$-mercaptoethanol (Biotech), $30 \mathrm{mg} / \mathrm{l}$ penicillin (Amresco), and $75 \mathrm{mg} / \mathrm{l}$ streptomycin (Amresco). One thousand iGSCs, FGSCs or SSCs were 3D co-cultured with $3 \times 10^{4}$ gonadal somatic cells. The co-cultures from 96-well U-bottom, low-binding culture plates were transferred onto transwell-COL membranes (Coaster) soaked in $\alpha$-MEM- -based medium, $\alpha$-MEM supplemented with $2 \%$ FBS, 2 mM L-glutamine, $150 \mu \mathrm{M}$ ascorbic acid (Sigma), $50 \mathrm{mM} \beta$-mercaptoethanol, 30 $\mathrm{mg} / \mathrm{lpenicillin}$, and $75 \mathrm{mg} / \mathrm{l}$ streptomycin. At 4 days of culture, the culture medium 
1 was changed to StemPro-34-based medium, StemPro-34 SFM (Life Technologies)

2 supplemented with $10 \%$ FBS, $2 \mathrm{mM}$ L-glutamine, $150 \mu \mathrm{M}$ ascorbic acid, 50

$3 \mathrm{mM} \beta$-mercaptoethanol, $30 \mathrm{mg} / \mathrm{l}$ penicillin, and $75 \mathrm{mg} / \mathrm{l}$ streptomycin. From 7 to 10

4 days of culture, $600 \mathrm{nM}$ ICI182780 was added to the StemPro-34-based medium. At

511 days of culture, the culture medium was changed to StemPro-34-based medium

6 without ICI182780. After 21 days of culture, individual follicles were manually

7 dissociated using sharpened tungsten needles.

8 Follicle 3D culture. The single follicles were cultured on transwell-COL membranes

9 with medium, $\alpha$-MEM supplemented with 5\% FBS, 2\% polyvinylpyrrolidone

(Sigma), $2 \mathrm{mM}$ L-glutamine, $150 \mu \mathrm{M}$ ascorbic acid, $50 \mathrm{mM} \beta$-mercaptoethanol, 30 $\mathrm{mg} / \mathrm{l}$ penicillin, $75 \mathrm{mg} / \mathrm{l}$ streptomycin, $30 \mathrm{mg} / \mathrm{ml}$ pyruvate (Amresco), $0.1 \mathrm{IU} / \mathrm{ml}$ follicle-stimulating hormone (FSH; MSD), $15 \mathrm{ng} / \mathrm{ml} \mathrm{BMP15,} \mathrm{and} 15 \mathrm{ng} / \mathrm{ml}$ GDF9 (R\&D Systems). At 2 days of culture, the culture medium was changed to medium without BMP15 and GDF9, and then follicles were incubated in $0.1 \%$ Type IV Collagenase (Invitrogen) for $5 \mathrm{~min}$. After washing with $\alpha$-MEM supplemented with $5 \%$ FBS several times, the follicles were cultured in medium without BMP15 and GDF9. After 14 days of culture, cumulus-oocyte complexes grown on the membrane were picked up by a fine glass capillary.

In vitro maturation, in vitro fertilization, and embryo transfer. The cumulus-oocyte complexes were cultured with $\alpha$-MEM containing 5\% FBS, $30 \mathrm{mg} / \mathrm{ml}$ pyruvate (Amresco), $0.1 \mathrm{IU} / \mathrm{ml} \mathrm{FSH,} 4 \mathrm{ng} / \mathrm{ml} \mathrm{EGF,} 1.2 \mathrm{IU} / \mathrm{ml} \mathrm{hCG}$ (gonadotropin, ASKA), $4 \mathrm{ng} / \mathrm{ml}$ bFGF, $30 \mathrm{mg} / \mathrm{l}$ penicillin, $75 \mathrm{mg} / \mathrm{l}$ streptomycin. After 17-20 hours of culture, mature oocytes with expanded cumulus cells were fertilized in HTF medium (SAGE) by sperm. Embryos developed to the 2-cell stage were transferred into the oviducts of pseudopregnant females at 0.5 day post-coitum.

\section{Data Availability}

Original data of Hi-C have been deposited in the Gene Expression Omnibus database (accession number: 135104). Original data of RNA-Seq have been deposited in the 
1 Gene Expression Omnibus database (accession number: 134727). Original data of 2 MeDIP-Seq have been deposited in the Gene Expression Omnibus database 3 (accession number: 134640). Original data of Microarrays have been deposited in the 4 Gene Expression Omnibus database (accession number: GSE38776). All other 5 relevant data are available from the corresponding author upon request.

6

21. Yoshimizu, T. et al. Germline-specific expression of the Oct-4/green fluorescent protein (GFP) transgene in mice. Dev Growth Differ41, 675-684 (1999).

22. Reddy, P. et al. Oocyte-specific deletion of Pten causes premature activation of the primordial follicle pool. Science319, 611-613 (2008).

23. Lan, Z. J., Xu, X. \& Cooney, A. J. Differential oocyte-specific expression of Cre recombinase activity in GDF-9-iCre, Zp3cre, and Msx2Cre transgenic mice. Biol Reprod71, 1469-1474 (2004).

24. Wu, J., Jester, W. F., Jr. \& Orth, J. M. Short-type PB-cadherin promotes survival of gonocytes and activates JAK-STAT signalling. Dev Biol284, 437-450 (2005).

25. Wu, J. et al. Short-type PB-cadherin promotes self-renewal of spermatogonial stem cells via multiple signaling pathways. Cell Signal20, 1052-1060 (2008).

26. Yuan, Z., Hou, R. \& Wu, J. Generation of mice by transplantation of an adult spermatogonial cell line after cryopreservation. Cell Prolif42, 123-131 (2009).

27. Zou, K. et al. Production of offspring from a germline stem cell line derived from neonatal ovaries. Nat Cell Biol11, 631-636 (2009).

28. Rohrborn, G. Frequencies of spontaneous non-disjunction in metaphase II. Oocytes of mice. Humangenetik 16, 123-125 (1972).

29. Eisen, M. B., Spellman, P. T., Brown, P. O. \& Botstein, D. Cluster analysis and display of genome-wide expression patterns. Proc Natl Acad Sci U S A95, 14863-14868 (1998).

30. Naumova, N. et al. Organization of the mitotic chromosome. Science342, 948-953 (2013).

31. Huang, L., Ma, F., Chapman, A., Lu, S. \& Xie, X. S. Single-Cell Whole-Genome Amplification and Sequencing: Methodology and Applications. Annu Rev Genomics Hum Genet16, 79-102 (2015).

32. Diaz, N. et al. Chromatin conformation analysis of primary patient tissue using a low input Hi-C method. Nat Commun9, 4938 (2018).

33. Ke, Y. et al. 3D Chromatin Structures of Mature Gametes and Structural Reprogramming during Mammalian Embryogenesis. Cell170, 367-381 e320 (2017).

34. Rao, S. S. et al. A 3D map of the human genome at kilobase resolution reveals principles of chromatin looping. Cell159, 1665-1680 (2014).

35. Servant, N. et al. HiC-Pro: an optimized and flexible pipeline for Hi-C data processing. Genome Biol16, 259 (2015).

36. Langmead, B. \& Salzberg, S. L. Fast gapped-read alignment with Bowtie 2. Nat Methods9, 357-359 (2012).

37. Imakaev, M. et al. Iterative correction of Hi-C data reveals hallmarks of chromosome organization. Nat Methods9, 999-1003 (2012).

38. Servant, N. et al. HiTC: exploration of high-throughput ' $\mathrm{C}$ ' experiments. Bioinformatics28, 2843-2844 (2012). 
39. Dixon, J. R. et al. Chromatin architecture reorganization during stem cell differentiation. Nature518, 331-336 (2015).

40. Pertea, M., Kim, D., Pertea, G. M., Leek, J. T. \& Salzberg, S. L. Transcript-level expression analysis of RNA-seq experiments with HISAT, StringTie and Ballgown. Nat Protoc11, 1650-1667 (2016).

41. Trapnell, C. et al. Differential gene and transcript expression analysis of RNA-seq experiments with TopHat and Cufflinks. Nat Protoc7, 562-578 (2012).

42. Huang da, W., Sherman, B. T. \& Lempicki, R. A. Systematic and integrative analysis of large gene lists using DAVID bioinformatics resources. Nat Protoc4, 44-57 (2009).

43. Su, G., Morris, J. H., Demchak, B. \& Bader, G. D. Biological network exploration with Cytoscape 3. Curr Protoc Bioinformatics47, 813 11-24 (2014).

44. Zhang, X. L. et al. Integrative epigenomic analysis reveals unique epigenetic signatures involved in unipotency of mouse female germline stem cells. Genome Biol17, 162 (2016).

45. Zhang, Y. et al. Model-based analysis of ChIP-Seq (MACS). Genome Biol9, R137 (2008).

46. Hikabe, O. et al. Reconstitution in vitro of the entire cycle of the mouse female germ line. Nature539, 299-303 (2016).

Acknowledgements This work was supported by the National Key Research and Development Program of China (2018YFC1003501, 2017YFA0504201), National Nature Science Foundation of China (81720108017), the National Major Scientific Instruments and Equipment Development Project, National Nature Science Foundation of China (61827814).

Author contributions H.L., X.L. and G.G.T. conducted all the major experiments, data analysis and wrote the manuscript; D.L. performed embryo transfer; C. H. carried out in situ Hi-C library generation using a low amount of cells; X.D. and W.X. were responsible for karyotype analysis; L.H, Y.Y., and H.W. were responsible for immunofluorescence and histological analysis of ovarian tissue; Q.L., A.J.C. and J.X. conducted Gdf9-Cre ${ }^{+}$and GFP transgenic mice study; X.Z. carried out MeDIP-seq and bioinformatics; J.W. initiated and supervised the entire project, conducted SSC and FGSC transplantation, analyzed data and wrote the manuscript.

Competing interests The authors declare no competing interests.

Materials \& Correspondence Correspondence and requests for materials should be addressed to J.W. 


\section{$1 \quad$ Figure legends}

2

Fig. 1: SSCs transdifferentiate into oocytes in the ovaries of POF recipients and GFP-expressing offspring are generated from the transplanted SSCs from pou5f1/GFP transgenic mice. a, SSCs were transplanted into the ovaries of POF recipient mice. I, II, Representative morphologies of the ovaries from recipients with (I) or without (II) SSC transplantations. III, Follicles containing GFP-positive (green) oocytes in recipient ovaries at 8 weeks after transplantation of pou5f1/GFP transgenic SSCs. IV, Oocytes in a wild-type ovary without a GFP signal. b, DNA fluorescence in situ hybridization for SRY. SRY was only localized in oocytes (green) derived from SSCs in ovary (I). Nuclei were counterstained with DAPI (blue) (II). c, Circos plot showing the coverage from the single cell exon sequencing as a histogram. Grey represents FGSCs; Red represents GV oocytes derived from SSCs in the ovary; Blue represents SSCs. d, Karyotype analysis of mature oocytes from POF recipient ovaries at 2 months after pou5f1/GFP transgenic SSC transplantation. I, II, Representative morphologies of mature oocytes derived from pou5f1/GFP transgenic SSCs (I) emitting GFP fluorescence (II) under UV light. III-V, Cytogenetic analysis by G-band staining showing that some mature oocytes from SSCs had a karyotype of 20, Y. III: An example of 20, Y in mature oocytes derived from pou5f1/GFP transgenic SSCs. Arrow indicates the Y chromosome. IV: Example of 20, $\mathrm{X}$ in mature oocytes derived from pou5f1/GFP transgenic SSCs. V: Representative karyotype (20, X) of wild-type mature oocytes. VI, PCR analysis of Sry. M, 100 bp DNA marker; lane 1, SSCs; lane 2, mature oocytes derived from pou5f1/GFP transgenic SSCs; lane 3, wild-type mature oocytes; lane 4, mock. e, Example of offspring from POF recipient mice transplanted with pou5f1/GFP transgenic SSCs (I) and an example of a Southern blot of tail DNA (II). Genomic DNA was digested with EcoRI. Marker sizes are indicated to the right of the blot. Lanes 1, 3, 5, and 7: transgenic mice; lanes 2, 4, 6, and 8: wild-type mice. f, SSLP analysis of parents and their offspring mice through SSLP markers. M: DNA marker; lane 1: donor SSCs; lane 2: female recipients (POF); lane 3: 
1 mated males (C57BL/6); lanes 4-11: offspring from eight corresponding recipients

2 females. Scale bars, $50 \mu \mathrm{m}$ (a I, III, IV), $100 \mu \mathrm{m}$ (a II), $25 \mu \mathrm{m}$ (b I, II), $10 \mu \mathrm{m}$ (d I,

3 II).

4

Fig. 2: Tracking of transplanted SSCs in recipient ovaries. a, Transplanted SSCs from pou5f1/GFP transgenic mice were monitored by confocal laser scanning microscopy at 2 hours, and 2, 3, 4, 5, 6, 9, 12, and 15 days after transplantation into recipient ovaries. b, Gene expression dynamics during oogenesis in transplanted cells at 4, 6, 9, and 15 days after transplantation. c, Dual immunofluorescence analysis of MVH and GFP expression in transplanted cells at 2, 3, 4, 5, 6, 9, 12, and 15 days after transplantation. Scale bars, $50 \mu \mathrm{m}$.

\section{Fig. 3: Reorganization of the chromosome structure during SSC conversion to} iGSCs. a, Contact matrices from chromosome 16 in SSCs, iGSCs, and FGSCs. b, First principal component (PC1) value and normalized Hi-C interaction heat maps at a $40 \mathrm{~kb}$ resolution in SSCs, iGSCs, and FGSCs. The PC1 value was used to indicate the A/B compartment status, where a positive $\mathrm{PC} 1$ value represents the A compartment (blue) and a negative value represents the B compartment (yellow). Dashed lines indicate TAD boundaries in SSCs. c, Hieratical clustering of PC1 values for the A/B compartment status in SSCs, iGSCs, and FGSCs. d, Expression of genes that changed compartment status ("A to B" or "B to A") or remained the same ("stable") compared with SSCs (P-value by Wilcoxon's test). e, IGV snapshot of Dppa3 (Stella) showing concordance between its expression and PC1 values. f, Relative MeDIP-seq signal that changed compartment status ("A to B" or "B to A") or remained the same ("stable") compared with SSCs (P-value by Wilcoxon's test). ***p<0.0001.

Fig. 4: Offspring production of ovarian organoids derived from iGSCs and FGSCs. a, Ovarian organoid formation and development. Representative ovarian organoids with a merge of bright field and fluorescence. I-III, Ovarian organoids or co-cultures with somatic cells of gonad and iGSCs at 3 days (I), 2 weeks (II), and 3 
1 weeks (III). IV-VI, Ovarian organoids or co-cultures with somatic cells of gonads and

2 FGSCs at 3 days (IV), 2 weeks (V), and 3 weeks (VI). VII-IX, Images of aggregates

3 formed by somatic cells of gonads and SSCs at 3 days (VII), 2 weeks (VIII), and 3

4 weeks (IX). b, Follicle growth in vitro. I-III, VII, Representative follicles isolated

5 from ovarian organoids formed by somatic cells of gonads and iGSCs at 0 days (I), 2

6 days (II), 7 days (III), and 11 days (VII). IV-VI, VIII, Representative follicles isolated

7 from ovarian organoids formed by somatic cells of gonads and FGSCs at 0 days (IV),

82 days (V), 7 days (VI), and 11 days (VIII). IX, X, Cumulus-oocytes complexes

9 derived from iGSCs (IX) and FGSCs (X) before in vitro maturation. c, Representative

10 views of each stage of meiotic prophase I during ovarian organoid development after

11 stained with anti-Sycp3 and - $\mathrm{H} 2 \mathrm{AX}$ antibodies. d, Mature oocytes derived from

12 iGSCs or FGSCs after in vitro maturation. e, Two-cell embryos derived from iGSCs

13 or FGSCs after in vitro fertilization. f, Representative offspring derived from iGSCs

14 or FGSCs. g, Offspring were identified by Southern blotting. Lanes 1-4, offspring

15 derived from iGSCs, lane W, wild-type mice, lanes 5-8, offspring derived from

16 FGSCs. f, Offspring were identified by fluorescence. Lanes 1-4, offspring derived

17 from iGSCs, lane W, wild-type mice, lanes 5-8, offspring derived from FGSCs. Scale

18 bars, $100 \mu \mathrm{m}$ (a), $20 \mu \mathrm{m}$ (b I-VI), $40 \mu \mathrm{m}$ (b VII-VIII), $50 \mu \mathrm{m}$ (b IX-X, d, e), $5 \mu \mathrm{m}$ (c). 
bioRxiv preprint doi: https://doi.org/10.1101/764472; this version posted September 10, 2019. The copyright holder for this preprint (which was
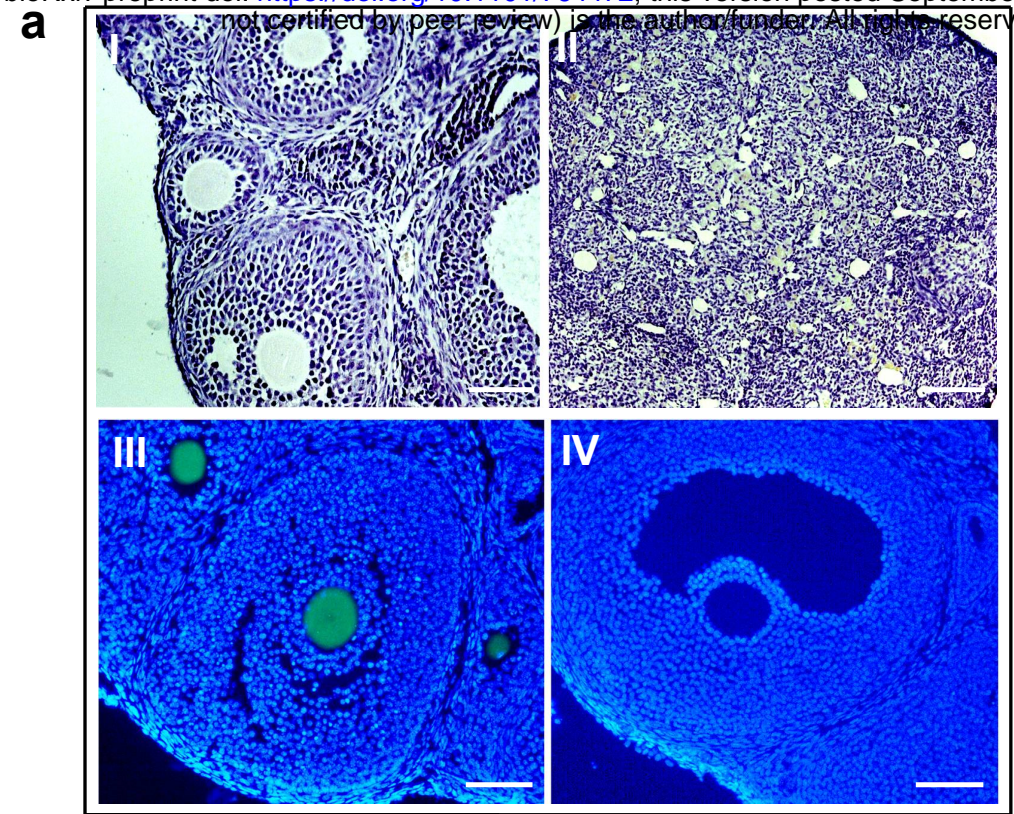

b
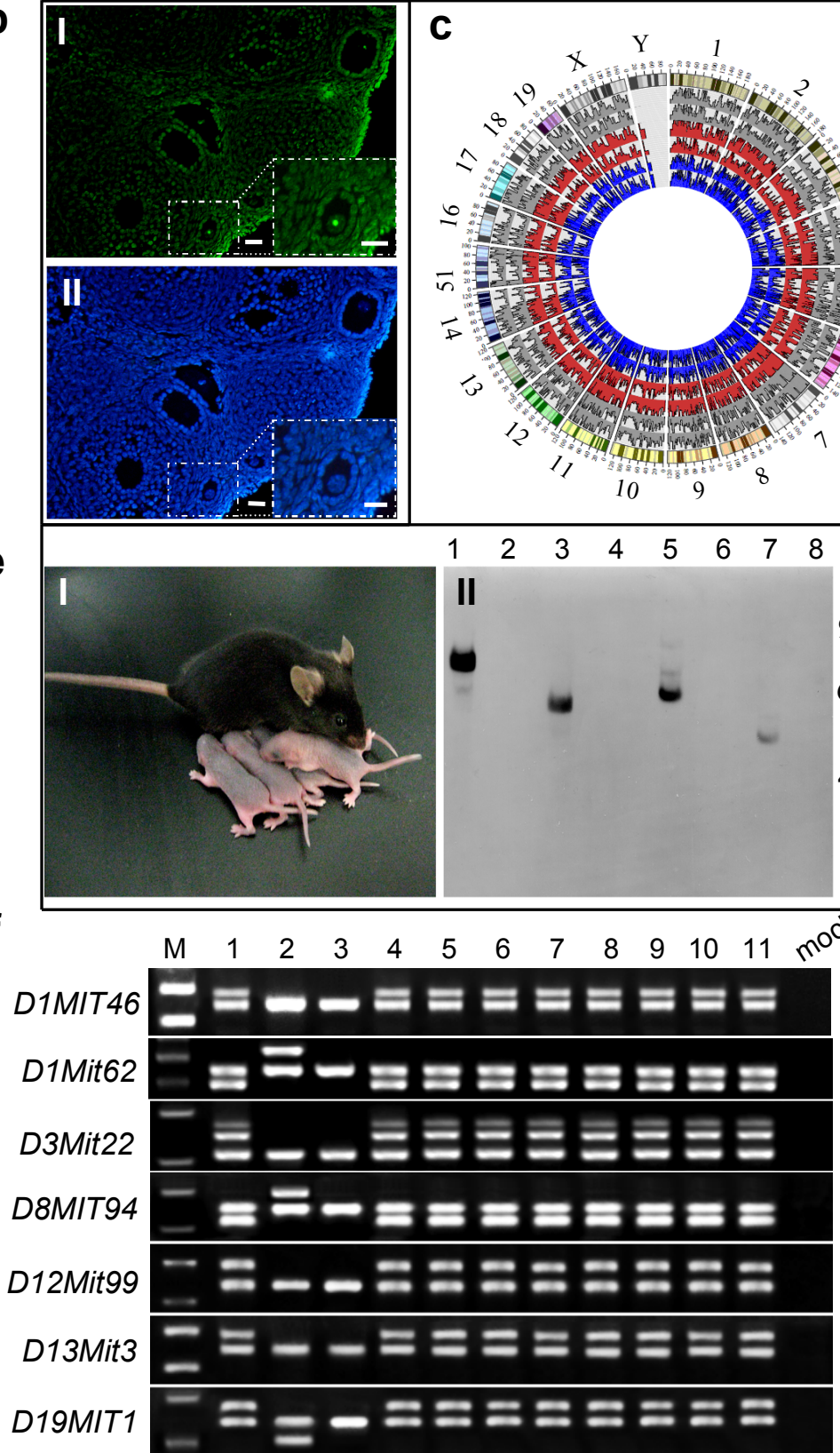

C
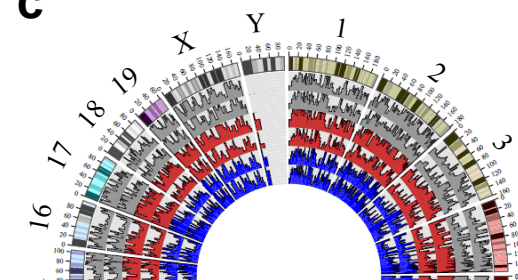

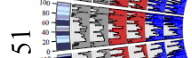
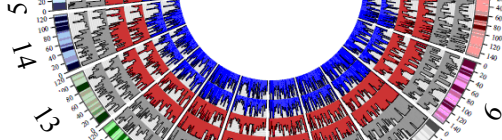
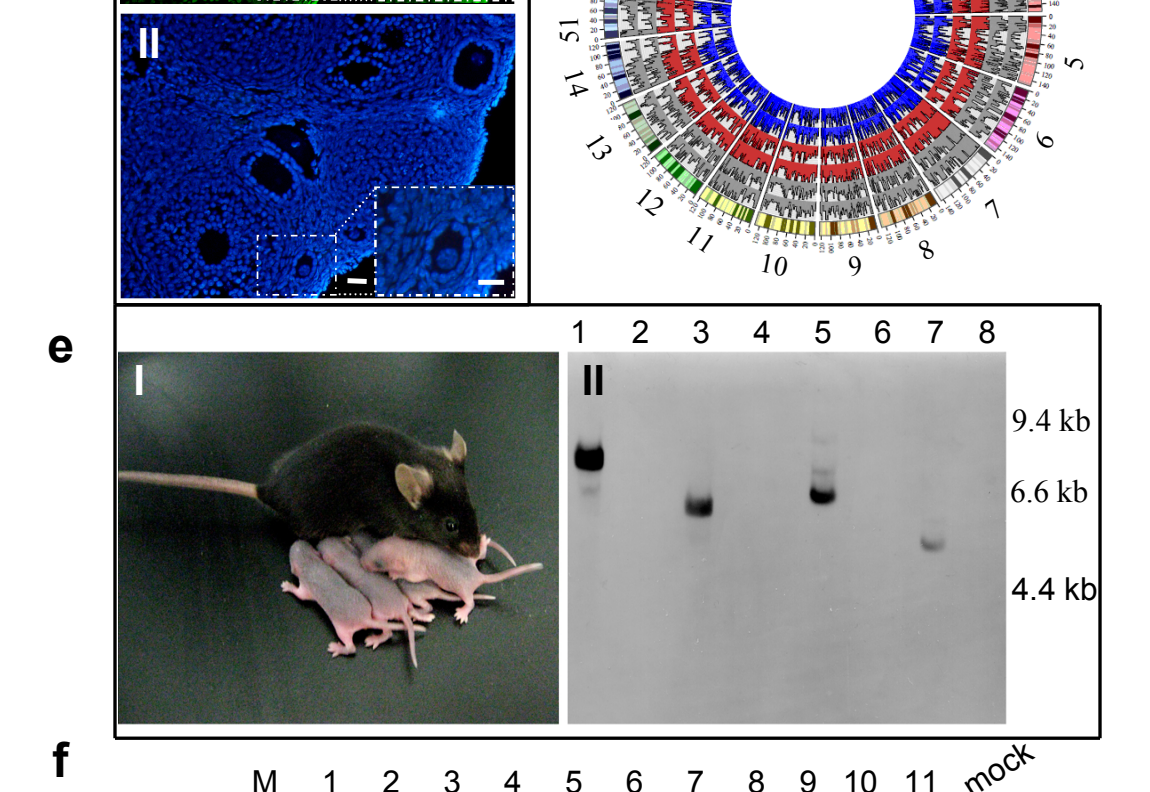

e

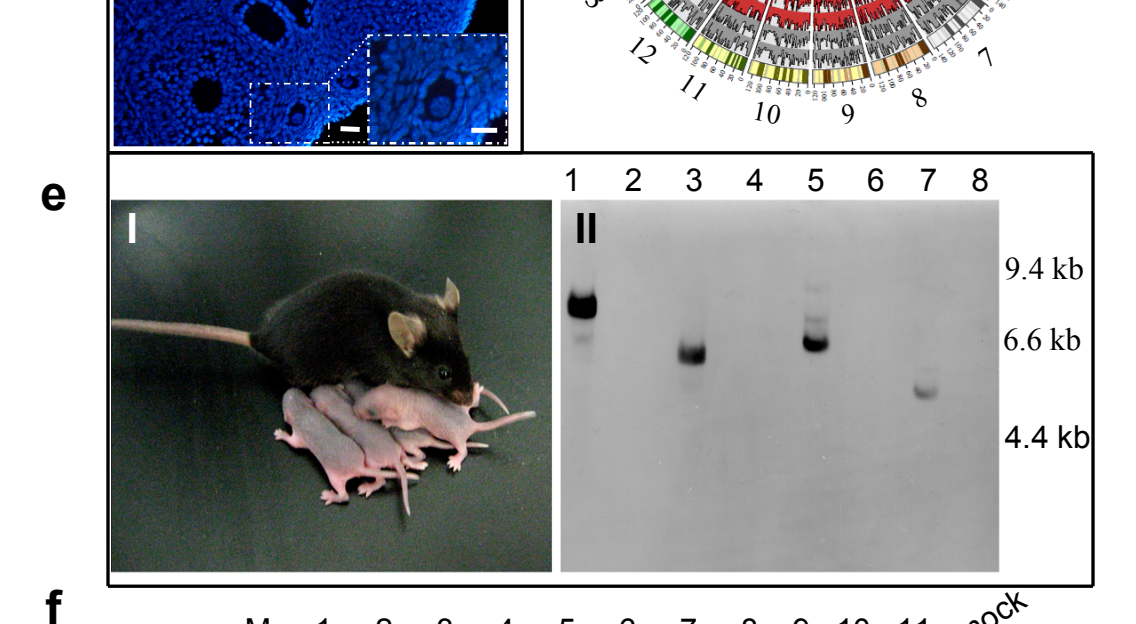

f

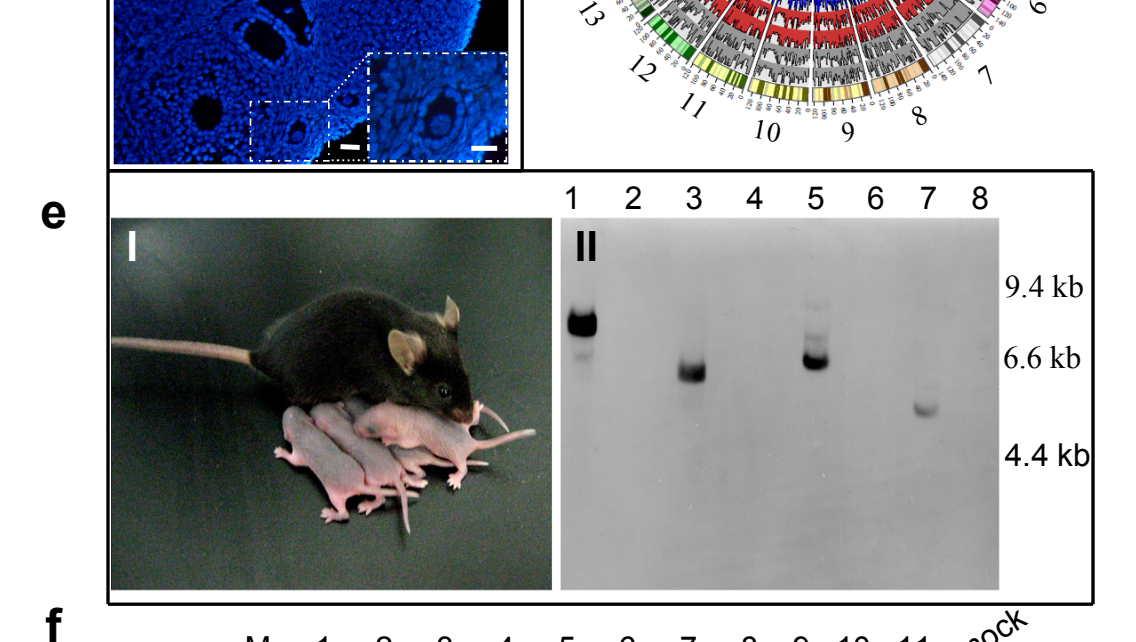

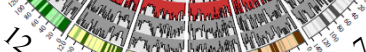

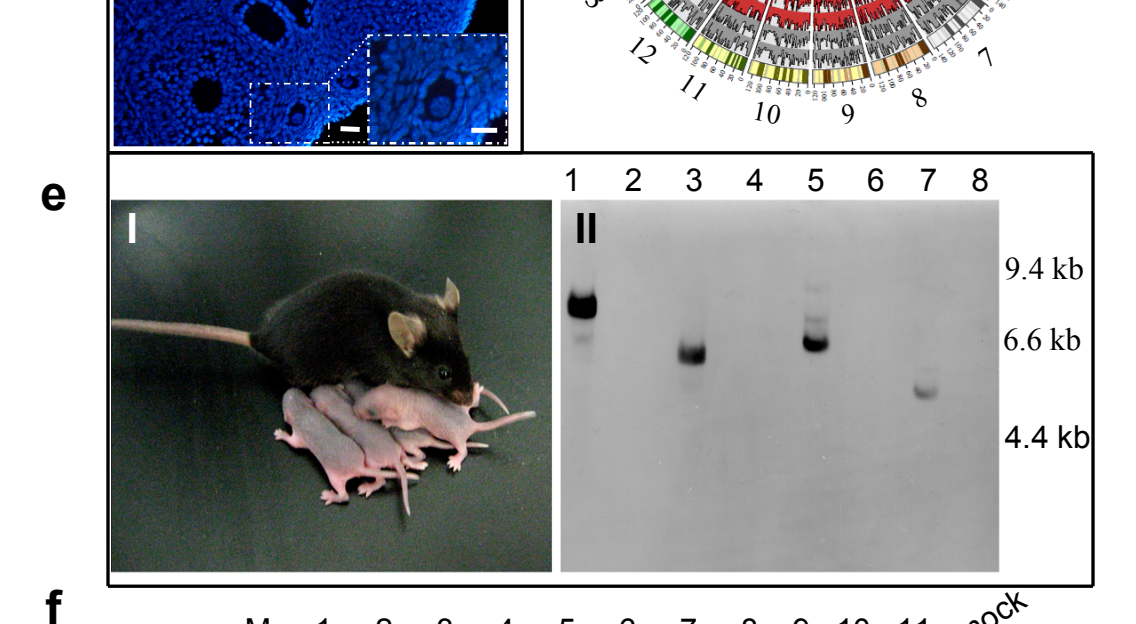

.

.
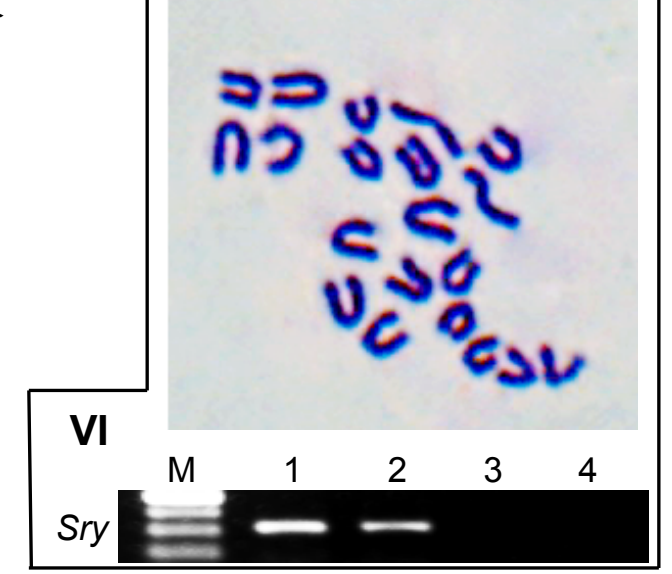


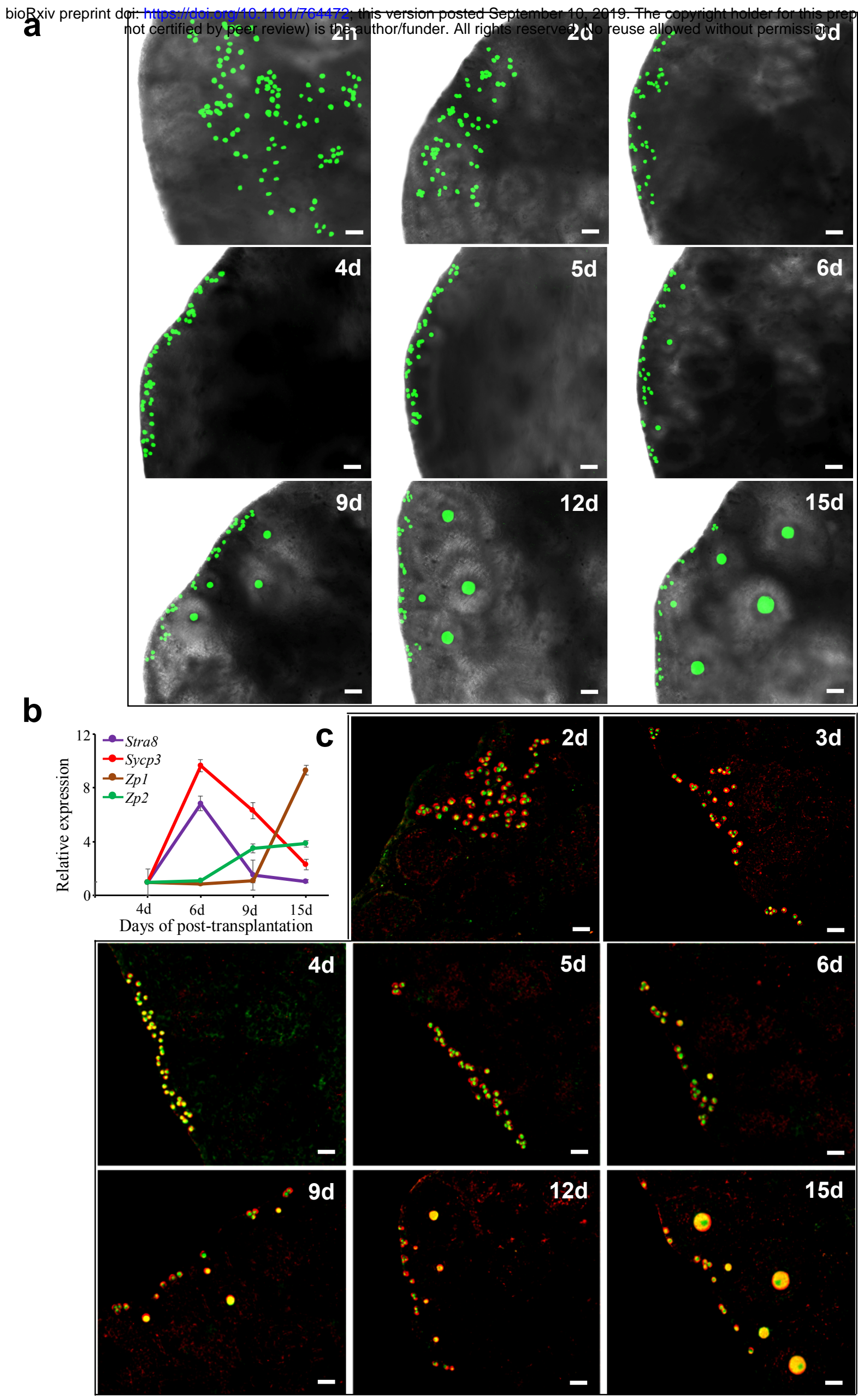


bioRxiv preprint doi: https://doi.org/10.1101/764472; this version posted September 10, 2019. The copyright holder for this preprint (which was

a Chromosome 16
not certified by peer review) is the author/

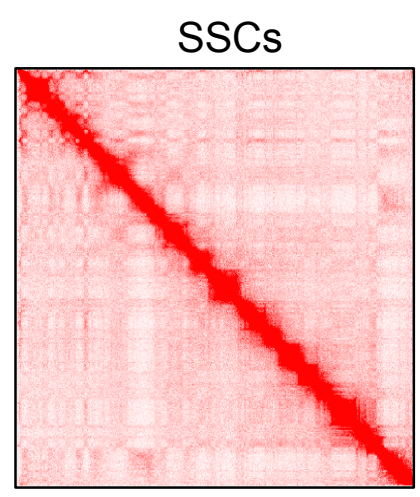

b
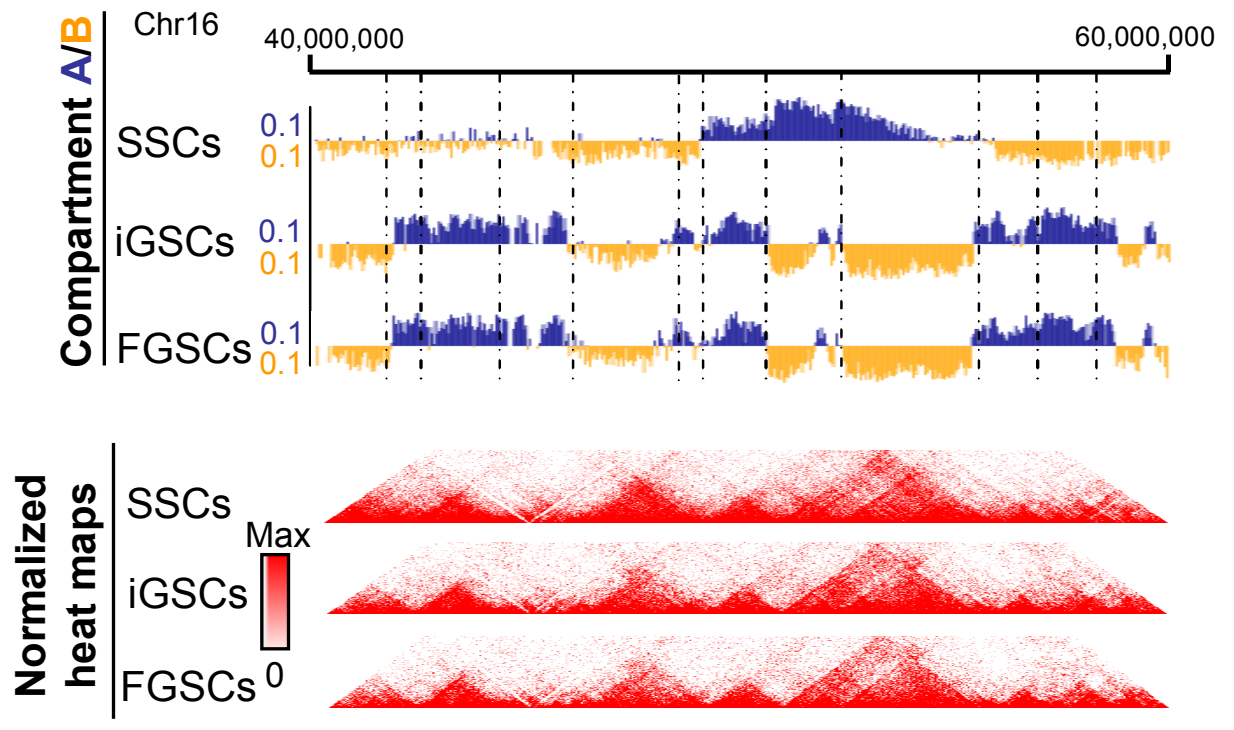

RefSeq genes
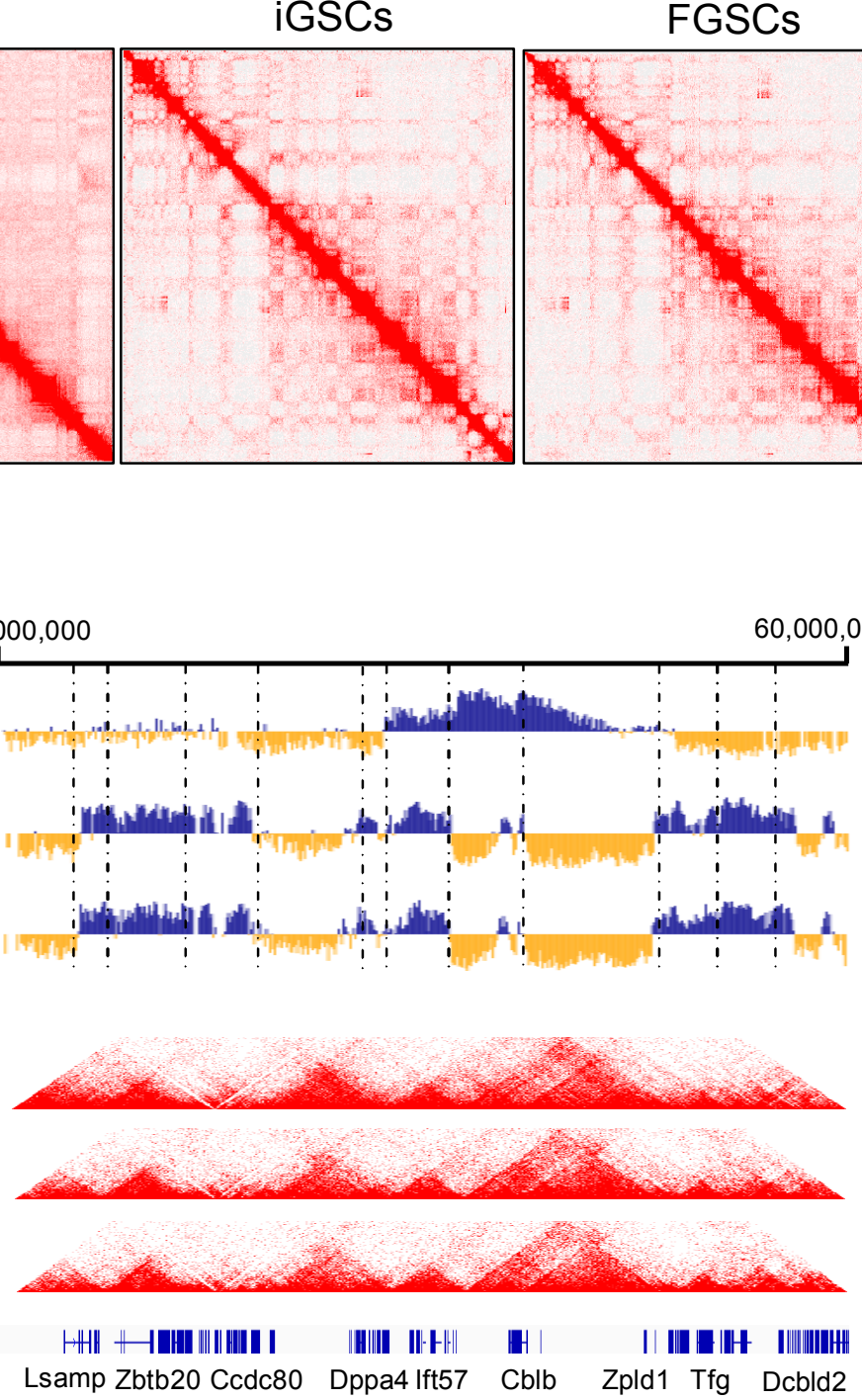

FGSCs

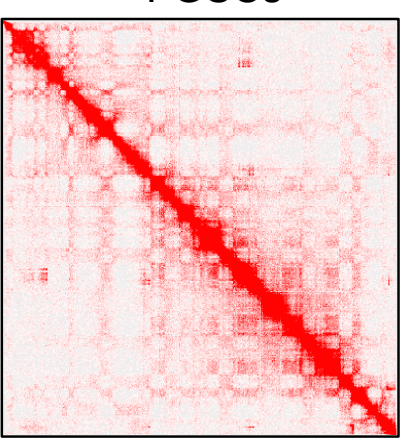

C

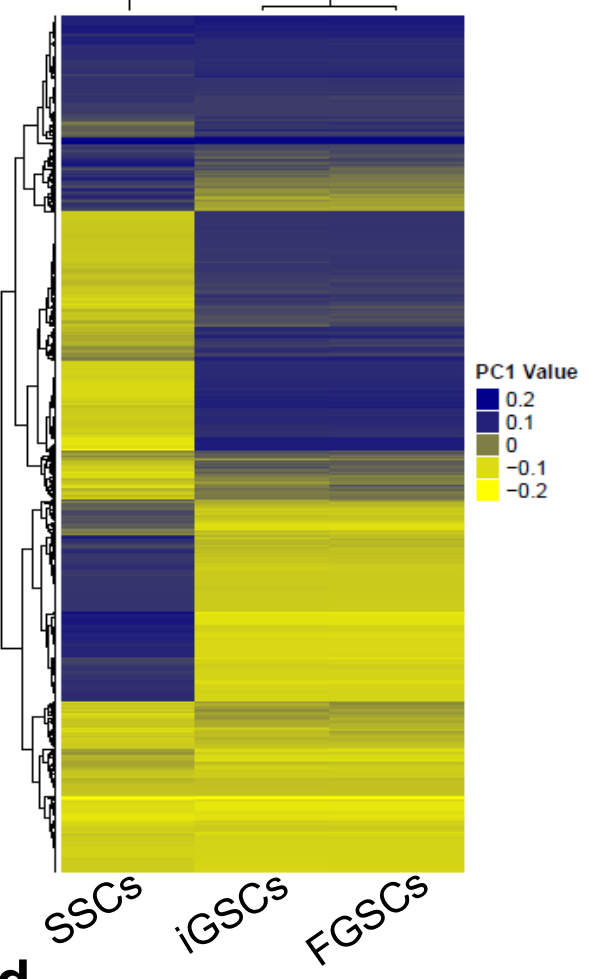

d

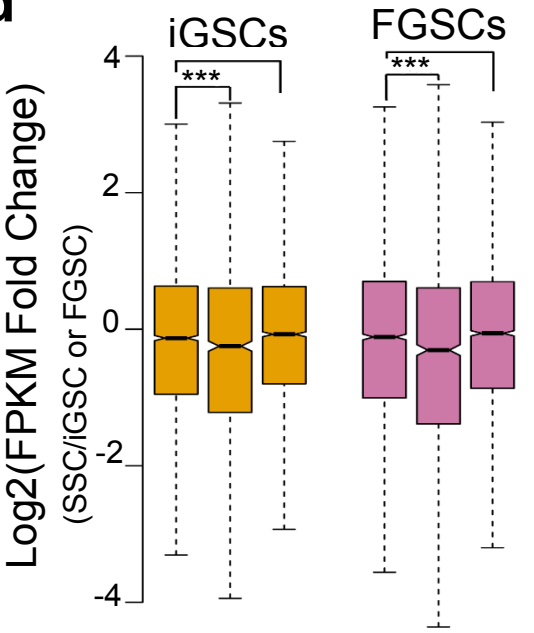

e

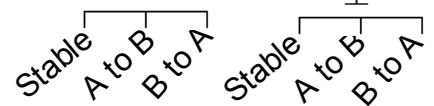
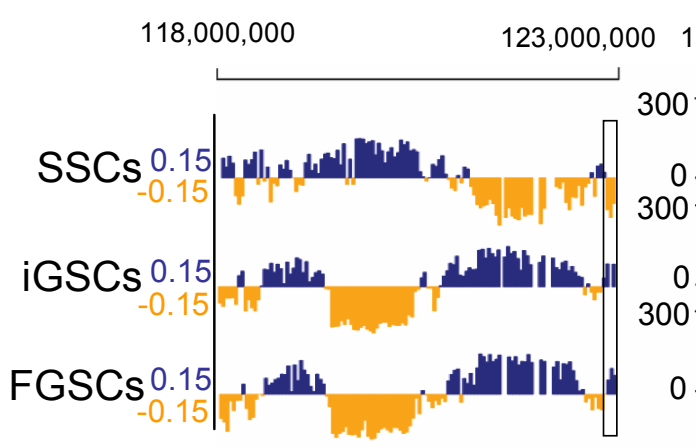

$122,585,000$

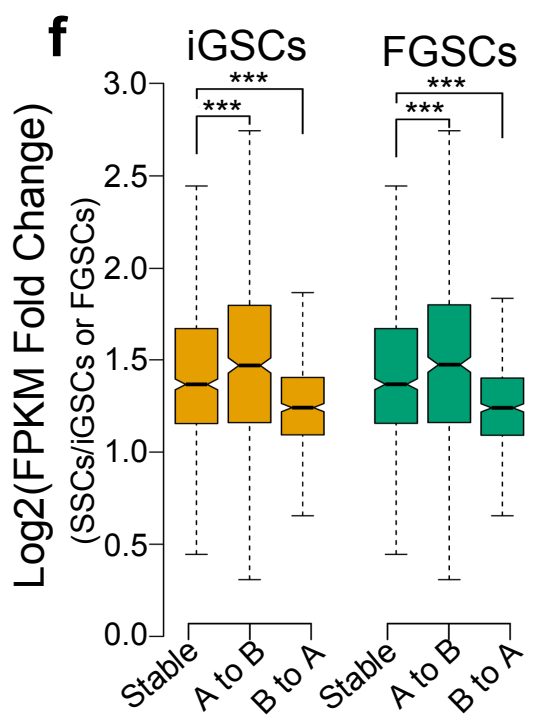


a

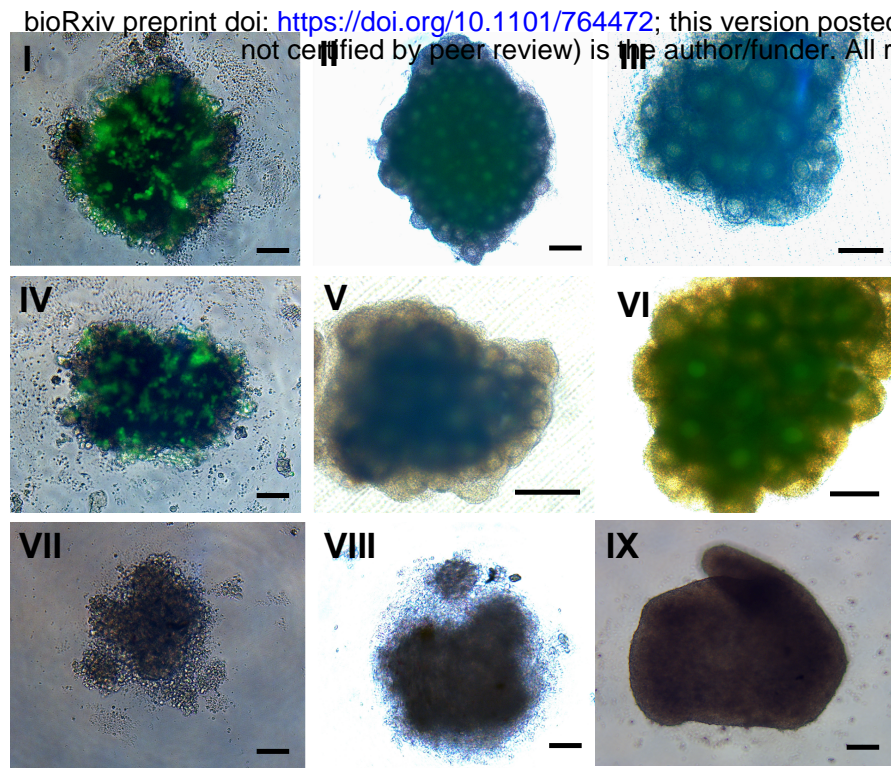

C

Leptotene

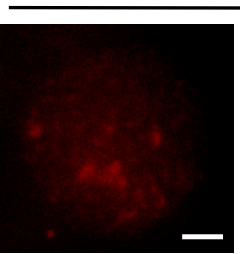

iGSCs

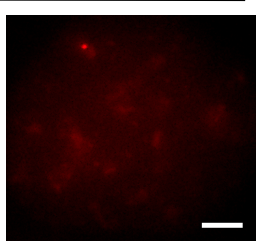

Zytotene
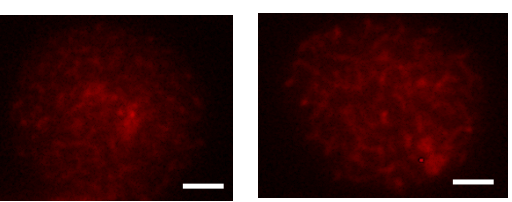

Pachytene

Pachytene
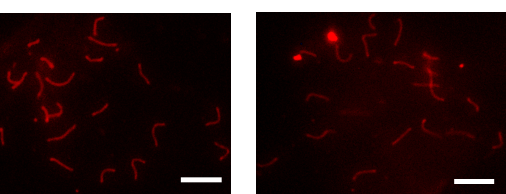

Diplotene

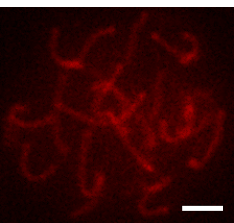

Sycp3

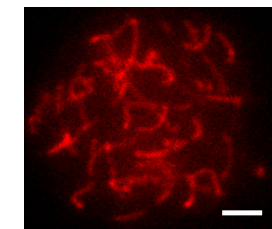

H2AX

d
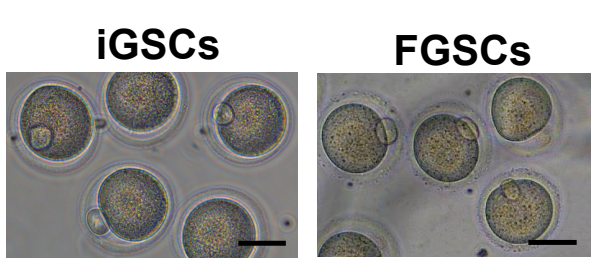

e

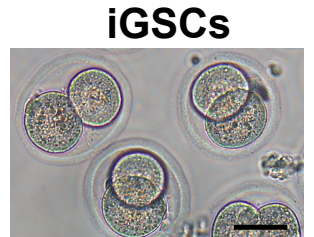

f

$\begin{array}{llllllllll}9 & 1 & 2 & 3 & 4 & W & 5 & 6 & 7 & 8\end{array}$
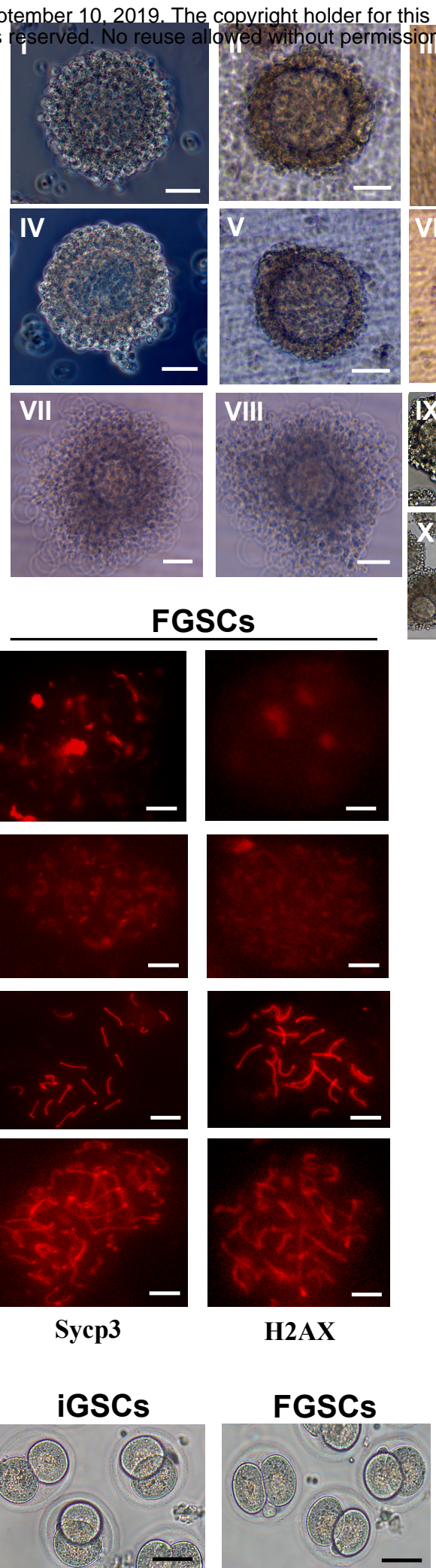

FGSCs
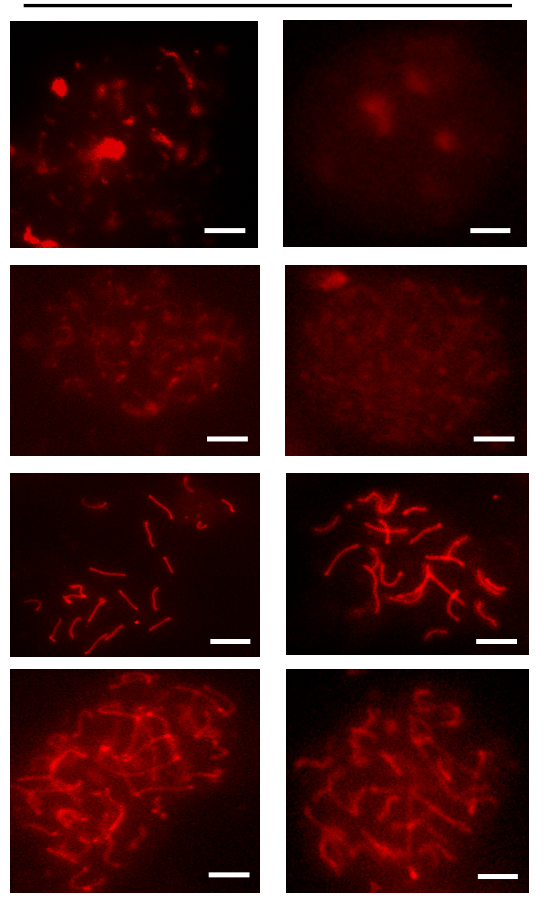

Sycp3

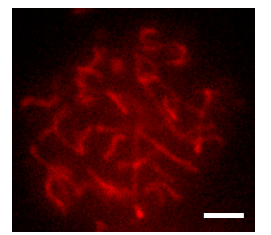

H2AX

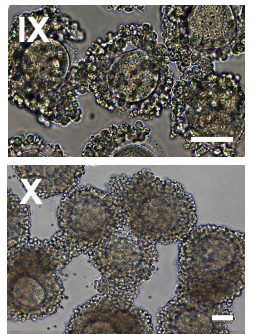

$\begin{array}{llllllllll}\text { h } & 1 & 2 & 3 & 4 & \text { W } & 5 & 6 & 7 & 8\end{array}$

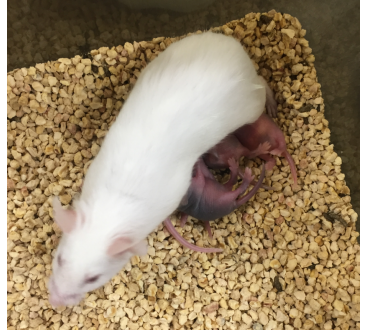

$9.4 \mathrm{~kb}$

$6.6 \mathrm{~kb}$

$--m-$

$4.4 \mathrm{~kb}$ 